\title{
Spin structures on loop spaces that characterize string manifolds
}

\author{
KONRAD WALDORF
}

\begin{abstract}
Classically, a spin structure on the loop space of a manifold is a lift of the structure group of the looped frame bundle from the loop group to its universal central extension. Heuristically, the loop space of a manifold is spin if and only if the manifold itself is a string manifold, against which it is well known that only the if part is true in general. In this article we develop a new version of spin structures on loop spaces that exists if and only if the manifold is string. This new version consists of a classical spin structure plus a certain fusion product related to loops of frames in the manifold. We use the lifting gerbe theory of Carey and Murray, recent results of Stolz and Teichner on loop spaces, and some of our own results about string geometry and Brylinski-McLaughlin transgression.
\end{abstract}

57R15; 58B05, 53C08

\section{Introduction}

The Witten genus was introduced by Witten [29], using supersymmetric sigma models with a spin manifold $M$ for a target space, and $S^{1}$-equivariant Dirac operators on the free loop space $L M$. Although the Witten genus is well defined (it is a power series in the Pontryagin numbers of $M$ ), the approach via loop space geometry still lacks a rigorous understanding.

Dirac operators can be considered on manifolds with a spin structure, ie with a lift of the structure group of the frame bundle to its universal covering group. The frame bundle of the loop space of an $n$-dimensional spin manifold $M$ is an $L \operatorname{Spin}(n)$-bundle. Killingback defined [8] a spin structure on $L M$ to be a lift of the structure group of the frame bundle of $L M$ to the universal central extension

$$
1 \rightarrow \mathrm{U}(1) \rightarrow \widetilde{L \operatorname{Spin}(n)} \rightarrow L \operatorname{Spin}(n) \rightarrow 1 \text {. }
$$

Killingback showed that the existence of spin structures on $L M$ is obstructed by a class $\lambda_{L M} \in \mathrm{H}^{3}(L M, \mathbb{Z})$. This class is often called the string class of $M$. In view of the following discussion, however, we'd better call it the spin class of $L M$. 
Killingback showed ${ }^{1}$ that the class $\lambda_{L M}$ is the transgression of the first fractional Pontryagin class $\frac{1}{2} p_{1}(M) \in \mathrm{H}^{4}(M, \mathbb{Z})$, ie the image of $\frac{1}{2} p_{1}(M)$ under the homomorphism

$$
\tau: \mathrm{H}^{4}(M, \mathbb{Z}) \rightarrow \mathrm{H}^{3}(L M, \mathbb{Z}), \quad x \mapsto \int_{S^{1}} \mathrm{ev}^{*} x,
$$

where ev: $S^{1} \times L M \rightarrow M$ is the evaluation map. See also Pilch and Warner [15], Carey and Murray [7] and McLaughlin [10].

Spin manifolds with vanishing first fractional Pontryagin class, $\frac{1}{2} p_{1}(M)=0$, are called string manifolds. Given the relation

$$
\tau\left(\frac{1}{2} p_{1}(M)\right)=\lambda_{L M}
$$

it is clear that the loop space of a string manifold is spin. In this article we are concerned with the converse proposition: is a manifold string when its loop space is spin? We recall three seminal results concerning this question. With methods of algebraic topology, McLaughlin showed the following.

Theorem 1.1 (McLaughlin [10, Theorem 3.1]) Suppose $M$ is a 2-connected spin manifold of dimension greater than 5. Then $L M$ is spin if and only if $M$ is string.

Similarly, but with more advanced methods using Hochschild cohomology, Kuribayashi and Yamaguchi proved that the assumption of 2-connectedness may be replaced by a condition that admits nontrivial $\pi_{2}$.

Theorem 1.2 (Kuribayashi and Yamaguchi [9, Theorem 1.2]) Let $M$ be a simply connected smooth manifold. Suppose $M$ is 4-dimensional, or $M$ has the structure of a compact homogenous space, or $M$ is a product of such spaces. Then $L M$ is spin if and only if $M$ is string.

In contrast to the previous two results, Pilch and Warner have shown that the assumption of simple connectedness cannot not be dropped (at least not when we consider a general principal $\operatorname{Spin}(n)$-bundle $P$ instead of the frame bundle).

Theorem 1.3 (Pilch and Warner [15, Section 3]) There exists a non-simply connected smooth manifold $M$ and a principal $\operatorname{Spin}(n)$-bundle $P$ over $M$ such that $\frac{1}{2} p_{1}(P) \neq 0$ and $L P$ is spin.

\footnotetext{
${ }^{1}$ In fact, Killingback defers the proof to a paper "in preparation" that I was not able to find. The earliest reference I know that contains a proof is [10].
} 
The situation that the loop space of a spin manifold $M$ is spin while $M$ is not string occurs evidently when the transgression homomorphism $\tau$ is not injective. Loosely speaking, the passage to the loop space loses information. The question is how this lost information can be restored on the loop space side. On a geometric level, this means to add additional structure to spin structures on loop spaces. To come back to the Dirac operators on the loop space: such operators could then be required to preserve this additional structure.

Witten [29] proposes that spin structures on the loop space have to be equivariant with respect to the rotation action of $S^{1}$ on $L M$, and accordingly considers $S^{1}$-equivariant Dirac operators. This leads to $S^{1}$-equivariant index theory on loop spaces; see eg Alvarez, Killingback, Mangano and Windey $[1 ; 2]$. The addition of $S^{1}$-equivariance, or more general, equivariance under the group $\mathcal{D i f f}^{+}\left(S^{1}\right)$ of orientation-preserving diffeomorphisms of $S^{1}$, indeed eliminates the counterexample of Theorem 1.3, as proved in Pilch and Warner [15]. In general, however, it is, to my best knowledge, not known whether a manifold is string if and only if its loop space has a $\operatorname{Diff}^{+}\left(S^{1}\right)$ equivariant spin structure.

One of the problems with $\mathcal{D i f f}^{+}\left(S^{1}\right)$-equivariance is that $\mathcal{D i f f}^{+}\left(S^{1}\right)$ is connected and hence acts separately on each connected component of $L M$. Assuming for a moment that $M$ is connected, these components are labeled by the fundamental group $\pi_{1}(M)$, so that the obstruction against lifting a spin structure on $L M$ to a $\operatorname{Diff}^{+}\left(S^{1}\right)$ equivariant spin structure splits into $\left|\pi_{1}(M)\right|$ many unrelated obstructions.

In this article, we introduce a new additional structure for spin structures on loop spaces. In particular, we establish a relation between the separate spin structures on different connected components of $L M$. This new additional structure is called a fusion product, and the corresponding spin structures are called fusion spin structures; see Definition 3.6. For the convenience of the reader, let me summarize the main idea behind fusion products.

In a more general context, the author [26] defined fusion products for U(1)-bundles over loop spaces. A fusion product defines a relation between the fibers of the bundle over the three loops emerging from three paths connecting a common initial point with a common end point. In particular, since these three loops may be elements of different connected components of the loop space, the existence of a fusion product cannot be explored separately over each connected component.

To apply the general concept of a fusion product to the present situation, we make two observations. The first is that the central extension $\overline{L \operatorname{Spin}(n)}$, considered as a $\mathrm{U}(1)$-bundle over $L \operatorname{Spin}(n)$, carries a canonical fusion product. In fact, we prove in Theorem 4.7 the existence of canonical fusion products for a large class of central 
extensions of loop groups. Our result includes the universal central extension of a compact, connected, simple and simply connected Lie group.

The second observation is that a spin structure on the loop space $L M$ can equivalently be understood as a $\mathrm{U}(1)$-bundle $\mathcal{S}$ over $L F M$, the loop space of the total space of the frame bundle of $M$, together with a certain action of $\overline{L \operatorname{Spin}(n)}$. Now, our new additional structure for spin structures on loop spaces is a fusion product on $\mathcal{S}$, subject to the condition that the $\widetilde{L \operatorname{Spin}(n)}$-action on $\mathcal{S}$ is compatible with the two fusion products on $\widetilde{L \operatorname{Spin}(n)}$ and $\mathcal{S}$; see Definition 3.6.

The main result of this article is that fusion products for spin structures sufficiently confine the before-mentioned loss of information in the transgression process.

Theorem 1.4 Let $M$ be a spin manifold of dimension $n=3$ or $n>4$. Then $M$ is string if and only if $L M$ is fusion spin.

This paper is organized as follows. Section 2 is complementary and concerns the problem of characterizing spin manifolds by orientations of loop spaces, as well as its solution by Stolz and Teichner. This problem is analogous to the one addressed in the present article but in "one degree lower". The content of Section 2 is not used in the main text, but might be useful for getting familiar with the topic.

Section 3 gives the definition of our new notion of fusion spin structures (Definition 3.6), and introduces and reviews the necessary material. In particular, we reveal the canonical fusion product on the central extension $\widehat{L \operatorname{Spin}(n)}$. For this, we use an explicit model of $\widehat{L \operatorname{Spin}(n)}$, introduced by Mickelsson [11] and motivated by conformal field theory.

The next two sections prepare the machinery for the proof of our main result. Section 4 is concerned with the loop space side. There we recast fusion spin structures in the context of lifting bundle gerbes. The theory of lifting bundle gerbes was invented by Murray [12] for ordinary central extensions of Lie groups. The main result of Section 4 is an extension of this theory to fusion extensions, ie to central extensions of loop groups by $\mathrm{U}(1)$ whose underlying principal bundle carries a fusion product. This section includes a new additional structure for bundle gerbes over loop spaces called an internal fusion product (Definition 4.13). Lifting bundle gerbes with internal fusion products serve as a bundle gerbe-theoretic setting for fusion spin structures.

In Section 5 we provide a similar, gerbe-theoretic setting for string structures, on the basis of my paper [28]. That is, we regard string structures as trivializations of a certain bundle 2-gerbe, namely the Chern-Simons 2-gerbe of Carey, Johnson, Murray, Stevenson and Wang [6]. As the main point in Section 5, we introduce a categorical version of the transgression homomorphism $\tau$, which takes a bundle 2 -gerbe over 
$M$ to a bundle gerbe over $L M$ with internal fusion product. We prove that the transgression of the Chern-Simons 2-gerbe gives the spin lifting bundle gerbe with its internal fusion product (Proposition 5.12). This geometrical transgression procedure establishes the relation between string structures on $M$ and fusion spin structures on $L M$. In Section 6 we assemble the pieces and prove Theorem 1.4.

I remark that we do not discuss the relation between the set of string structures on $M$ and the set of fusion spin structures on $L M$. Although (according to Theorem 1.4) one set is empty if and only if the other is empty, fusion spin structures are still not good enough to achieve a bijection between the two sets. Such bijection is the subject of ongoing research, and will additionally employ a certain equivariance under thin homotopies of loops. As a consequence, we can - at the moment - not provide any new insights into the theory of Dirac operators on the loop space.

Acknowledgements This work is supported by the Deutsche Forschungsgemeinschaft (DFG) within the scientific network "String Geometry" (project code 594335). I thank the Department of Mathematics at Hamburg University for kind hospitality and support during the summer term. Many thanks to Ulrich Bunke, André Henriques, Thomas Nikolaus, Ulrich Pennig, Peter Teichner, Chris Schommer-Pries, Urs Schreiber, and Christoph Wockel for helpful questions, comments, and discussions.

\section{Spin manifolds and loop space orientations}

Analogous to the question how spin structures on loop spaces can characterize string manifolds is the question how orientations of loop spaces can characterize spin manifolds. This question was considered early by Atiyah [3] and recently solved by Stolz and Teichner [20]. What follows is a sketch of their argument.

There is a canonical double covering $\mathcal{O}_{L M}$ of $L M$, the orientation bundle of the loop space, whose construction we now recall: The monodromy in the central extension

$$
1 \rightarrow \mathbb{Z}_{2} \rightarrow \operatorname{Spin}(n) \rightarrow \mathrm{SO}(n) \rightarrow 1
$$

is a smooth map $m: L S O(n) \rightarrow \mathbb{Z}_{2}$. Taking free loops in the oriented frame bundle $F M$ of $M$ produces an $L S O(n)$-bundle $L F M$ over $L M$, and $\mathcal{O}_{L M}$ is obtained by extending the structure group of $L F M$ along $m$, ie

$$
\mathcal{O}_{L M}:=L F M \times_{L S O}(n) \mathbb{Z}_{2} .
$$

Accordingly, an orientation of $L M$ is defined to be a section of $\mathcal{O}_{L M}$. 
On the level of cohomology classes, the class in $\mathrm{H}^{1}\left(L M, \mathbb{Z}_{2}\right)$ of $\mathcal{O}_{L M}$ is the transgression of the second Stiefel-Whitney class $w_{2}(M) \in \mathrm{H}^{2}\left(M, \mathbb{Z}_{2}\right)$, ie the image of $w_{2}(M)$ under the $\mathbb{Z}_{2}$-reduced transgression homomorphism

$$
\tau: \mathrm{H}^{2}\left(M, \mathbb{Z}_{2}\right) \rightarrow \mathrm{H}^{1}\left(L M, \mathbb{Z}_{2}\right) .
$$

In particular, $L M$ is orientable if $M$ is spin.

In [3], Atiyah remarked that for a simply connected manifold $M$ the converse statement is true. Thus a simply connected manifold $M$ is spin if and only if $L M$ is orientable. This statement was later proved in detail by McLaughlin [10, Proposition 2.1].

The problem of characterizing nonsimply connected spin manifolds was solved by Stolz and Teichner [20]. Using methods of spin geometry, they recognized a crucial additional structure on the orientation bundle $\mathcal{O}_{L M}$, a so-called fusion product. Accordingly, among all orientations of $L M$, there is a subclass consisting of fusion-preserving orientations. Stolz and Teichner showed:

Theorem 2.1 [20, Theorem 9] Let $M$ be an oriented Riemannian manifold. Then there is a bijection

$$
\left\{\begin{array}{l}
\text { Equivalence classes of } \\
\text { spin structures on } M
\end{array}\right\} \cong\left\{\begin{array}{l}
\text { Fusion-preserving } \\
\text { orientations of } L M
\end{array}\right\} .
$$

In particular, $M$ is spin if and only if $L M$ is fusion orientable.

Speaking in terms of the framework used in the present article, the bijection of Theorem 2.1 is induced by a version of the Brylinski-McLaughlin transgression functor for abelian gerbes; see [26, Section 1.2] for a complete discussion.

The notion of a fusion product is crucial for the new version of spin structures we introduce in this article, and is explained in the following section.

\section{Fusion spin structures}

In this section we explain our new version of spin structures on loop spaces, which we call fusion spin structure; see Definition 3.6. We consider a spin manifold $M$ of dimension $n=3$ or $n>4$. In these dimensions, the group $\operatorname{Spin}(n)$ is simple, connected, simply connected and compact. We fix a generator $\gamma_{\text {can }} \in \mathrm{H}^{3}(\operatorname{Spin}(n), \mathbb{Z}) \cong \mathbb{Z}$. The loop group $L \operatorname{Spin}(n)$ has a universal central extension [16, Section 4.4]

$$
1 \longrightarrow \mathrm{U}(1) \longrightarrow \widetilde{L \operatorname{Spin}(n)} \stackrel{p}{\longrightarrow} L \operatorname{Spin}(n) \longrightarrow 1 \text {. }
$$


This universal extension is determined up to a sign, which we fix by requiring the following identity for its first Chern class (when considered as a principal U(1)-bundle over $L \operatorname{Spin}(n))$ :

$$
\tau\left(\gamma_{\text {can }}\right)+\mathrm{c}_{1}(\widetilde{L \operatorname{Spin}(n)})=0 \in \mathrm{H}^{2}(L \operatorname{Spin}(n), \mathbb{Z}) .
$$

This sign convention is opposite to the one of Pressley and Segal; see [16, Proposition 4.4.4 and 4.5.6]. We also remark that the transgression homomorphism $\tau$ is an isomorphism for simply connected Lie groups; see the discussion after [16, Equation 4.4.3].

We start by reviewing the classical notion of spin structures on loop spaces. We denote by $F M$ the spin-oriented frame bundle of $M$. It is a smooth $\operatorname{Spin}(n)$-principal bundle over $M$. Since $\operatorname{Spin}(n)$ is connected, $L F M$ is a Fréchet principal $L \operatorname{Spin}(n)$-bundle over $L M$; see [23, Lemma 5.1] and [17, Proposition 1.9].

Definition 3.1 [8] A spin structure on $L M$ is a lift of the structure group of the looped frame bundle $L F M$ from $L \operatorname{Spin}(n)$ to the central extension $\widetilde{L \operatorname{Spin}(n)}$.

Thus, a spin structure on $L M$ is a pair $(\mathcal{S}, \sigma)$ of a Fréchet principal $\widetilde{L \operatorname{Spin}(n)}$-bundle $\mathcal{S}$ over $L M$ together with a smooth map $\sigma: \mathcal{S} \rightarrow L F M$ such that the diagram

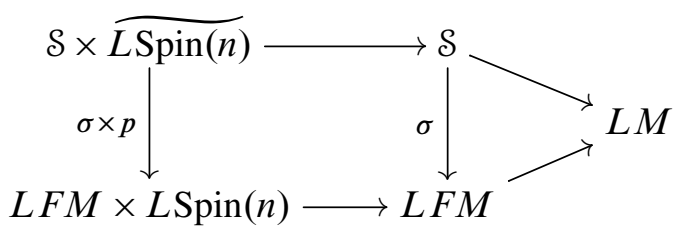

is commutative. A morphism between spin structures $\left(\mathcal{S}_{1}, \sigma_{1}\right)$ and $\left(\mathcal{S}_{2}, \sigma_{2}\right)$ is a smooth bundle morphism $\varphi: \mathcal{S}_{1} \rightarrow \mathcal{S}_{2}$ such that $\sigma_{1}=\sigma_{2} \circ \varphi$. The following lemma states an obvious general fact in lifting theory, which we need later.

Lemma 3.2 Suppose $(\mathcal{S}, \sigma)$ is a spin structure on $L M$. Define a right $\mathrm{U}(1)$-action on $\mathcal{S}$ by

$$
\mathcal{S} \times \mathrm{U}(1) \rightarrow \mathcal{S}, \quad(q, z) \mapsto q \cdot i\left(z^{-1}\right) .
$$

Here $i: \mathrm{U}(1) \rightarrow \widetilde{\operatorname{LSpin}(n)}$ is the inclusion of the central subgroup, and $\cdot$ is the principal $\widehat{L \operatorname{Spin}(n)}$-action on $\mathcal{S}$. Under this action, $\sigma: \mathcal{S} \rightarrow L F M$ is a principal Fréchet $\mathrm{U}(1)-$ bundle.

An important role in this article is played by so-called fusion in loop spaces. Fusion is a constraint for structure on a loop space $L X$ that is characteristically satisfied for structure obtained via transgression from $X$. This will be explained in Section 4.2. 
In the following we explain in detail the notion of a fusion product on a principal bundle over $L X$. We start with some technical preliminaries about paths. Let $X$ be a connected smooth manifold. By $P X$ we denote the set of paths in $X$ with "sitting instants", ie smooth maps $\gamma:[0,1] \rightarrow X$ that are locally constant near the endpoints. One can show that the set $P X$ is not a manifold, and in the following we will only treat it as a set. We denote by $P X^{[k]}$ the $k$-fold fiber product of $P X$ over the evaluation map ev: $P X \rightarrow X \times X$, ie the set of $k$-tuples of paths with a common initial point and a common end point. We have a map

$$
\cup: P X^{[2]} \rightarrow L X, \quad\left(\gamma_{1}, \gamma_{2}\right) \mapsto \overline{\gamma_{2}} \star \gamma_{1},
$$

where $\star$ denotes the path concatenation, and $\bar{\gamma}$ denotes the reversed path. This map is well-defined (it produces smooth loops) due to the sitting instants of the paths.

Definition 3.3 [26, Definition 2.1.3] Let $A$ be an abelian Lie group, and let $\mathcal{E}$ be a Fréchet principal $A$-bundle over $L X$. A fusion product on $\mathcal{E}$ assigns to each triple $\left(\gamma_{1}, \gamma_{2}, \gamma_{3}\right) \in P X^{[3]}$ a smooth, $A$-equivariant map

$$
\lambda_{\gamma_{1}, \gamma_{2}, \gamma_{3}}: \mathcal{E}_{\gamma_{1} \cup \gamma_{2}} \otimes \mathcal{E}_{\gamma_{2} \cup \gamma_{3}} \rightarrow \mathcal{E}_{\gamma_{1} \cup \gamma_{3}},
$$

such that the following two conditions are satisfied:

(i) Associativity For $\left(\gamma_{1}, \gamma_{2}, \gamma_{3}, \gamma_{4}\right) \in P X^{[4]}$ and $q_{i j} \in \mathcal{E}_{\gamma_{i} \cup \gamma_{j}}$,

$$
\lambda_{\gamma_{1}, \gamma_{3}, \gamma_{4}}\left(\lambda_{\gamma_{1}, \gamma_{2}, \gamma_{3}}\left(q_{12} \otimes q_{23}\right) \otimes q_{34}\right)=\lambda_{\gamma_{1}, \gamma_{2}, \gamma_{4}}\left(q_{12} \otimes \lambda_{\gamma_{2}, \gamma_{3}, \gamma_{4}}\left(q_{23} \otimes q_{34}\right)\right) .
$$

(ii) Smoothness If $U$ is a smooth manifold and $c: U \rightarrow P X^{[3]}$ is a map for which the three induced maps $e_{i j}:=\cup \circ \mathrm{pr}_{i j} \circ c: U \rightarrow L X$ are all smooth, then

$$
\lambda_{c}: e_{12}^{*} \mathcal{E} \otimes e_{23}^{*} \mathcal{E} \rightarrow e_{13}^{*} \mathcal{E}
$$

is a smooth morphism between bundles over $U$.

Given a principal $A$-bundle $\mathcal{E}$ over $L X$ with fusion product, one can construct a bundle gerbe over $X$ whose transgression is $\mathcal{E}$. It has the surjective submersion $\mathrm{ev}_{1}: P X \rightarrow X$, and the fusion product provides the bundle gerbe product. See Section 4.2 for more details.

I remark that in my papers $[25 ; 26 ; 27]$ I have treated the smoothness of fusion products by regarding $P X$ as a diffeological space. The above definition is equivalent to that treatment, but completely avoids diffeological spaces.

In the present article, fusion products are considered for two cases of principal U(1)bundles over loop spaces. The first case is the principal U(1)-bundle $\mathcal{S}$ over $L F M$ 
associated to a spin structure on $L M$ via Lemma 3.2. The second case is the underlying principal bundle of a central extension of the loop group $L G$ of a Lie group $G$. Our definition of a fusion spin structure (Definition 3.6 below) then combines both cases.

We shall first explain how a fusion product on a central extension of a loop group is required to be compatible with the group structure of the central extension.

Definition 3.4 Let $G$ be a Lie group and let $A$ be an abelian Lie group. A fusion extension of $L G$ by $A$ is a central extension

$$
1 \rightarrow A \rightarrow \mathcal{G} \rightarrow L G \rightarrow 1
$$

of Fréchet Lie groups, together with a multiplicative fusion product $\lambda$ on the principal $A$-bundle $\mathcal{G}$.

Here, a multiplicative fusion product satisfies

(4) $\lambda_{\gamma_{1}, \gamma_{2}, \gamma_{3}}\left(q_{12} \otimes q_{23}\right) \cdot \lambda_{\gamma_{1}^{\prime}, \gamma_{2}^{\prime}, \gamma_{3}^{\prime}}\left(q_{12}^{\prime} \otimes q_{23}^{\prime}\right)=\lambda_{\gamma_{1} \gamma_{1}^{\prime}, \gamma_{2} \gamma_{2}^{\prime}, \gamma_{3} \gamma_{3}^{\prime}}\left(q_{12} q_{12}^{\prime} \otimes q_{23} q_{23}^{\prime}\right)$

for all elements $q_{i j} \in \mathcal{G}_{\gamma_{i} \cup \gamma_{j}}$ and $q_{i j}^{\prime} \in \mathcal{G}_{\gamma_{i}^{\prime} \cup \gamma_{j}^{\prime}}$ and all $\left(\gamma_{1}, \gamma_{2}, \gamma_{3}\right),\left(\gamma_{1}^{\prime}, \gamma_{2}^{\prime}, \gamma_{3}^{\prime}\right) \in P G^{[3]}$.

Fusion extensions occur in the present article because of the following fact:

Theorem 3.5 The universal central extension $\widetilde{\operatorname{LSpin}(n)}$ is a fusion extension (in a canonical way).

We will give two proofs of this theorem. The first proof is given in the next paragraphs: we construct an explicit model $\mathcal{L}$ for the central extension $\overline{L \operatorname{Spin}(n)}$ and exhibit its fusion product. The second proof appears in Section 4.3 as Corollary 4.8. There we describe a construction of the central extension by transgression of the basic gerbe over $\operatorname{Spin}(n)$. A central result of my articles $[25 ; 26 ; 27]$ is that fusion products are a characteristic feature of structure in the image of transgression; this explains Theorem 3.5 in a conceptual way. In Section 4.3 we also show that the two constructions of $\widetilde{\operatorname{LSpin}(n)}$ are canonically isomorphic (Proposition 4.10).

Our explicit model $\mathcal{L}$ is motivated by conformal field theory and was introduced by Mickelsson [11]. It exists for any 2-connected Lie group $G$, such as $\operatorname{Spin}(n)$. We consider pairs $(\phi, z)$, where $\phi: D^{2} \rightarrow G$ is a smooth map and $z \in \mathrm{U}(1)$. For technical reasons, we require $\phi$ to be radially constant near the boundary, ie there exists $\epsilon>0$ such that $\phi\left(r \mathrm{e}^{2 \pi \mathrm{i} \varphi}\right)=\phi\left(\mathrm{e}^{2 \pi \mathrm{i} \varphi}\right)$ for all $1-\epsilon<r \leq 1$. On the set of pairs we impose the equivalence relation

$$
(\phi, z) \sim\left(\phi^{\prime}, z^{\prime}\right) \Leftrightarrow \partial \phi=\partial \phi^{\prime} \text { and } z=z^{\prime} \cdot \mathrm{e}^{2 \pi \mathrm{i} S_{\mathrm{WZ}}(\Phi)} .
$$


Here $\partial \phi$ denotes the restriction of $\phi$ to the boundary, and $\Phi: S^{2} \rightarrow G$ is the map defined on the northern hemisphere by $\phi$ (with the orientation-preserving identification) and on the southern hemisphere by $\phi^{\prime}$ (with the orientation-reversing identification). Since $\phi$ and $\phi^{\prime}$ are radially constant, this gives a smooth map. The symbol $S_{\mathrm{WZ}}$ stands for the Wess-Zumino term defined as follows. Because $G$ is 2-connected, the map $\Phi$ can be extended to a smooth map $\widetilde{\Phi}: D^{3} \rightarrow G$ defined on the solid ball. Then

$$
S_{\mathrm{WZ}}(\Phi):=\int_{D^{3}} \tilde{\Phi}^{*} H \quad \text { with } H:=\frac{1}{6}\langle\theta \wedge[\theta \wedge \theta]\rangle \in \Omega^{3}(G) .
$$

Here, $\theta \in \Omega^{1}(G, \mathfrak{g})$ is the left-invariant Maurer-Cartan from on $G$. The bilinear form $\langle-,-\rangle$ is the Killing form on the Lie algebra $\mathfrak{g}$, normalized such that the closed 3-form $H$ represents the fixed generator $\gamma_{\text {can }} \in \mathrm{H}^{3}(G, \mathbb{Z})$. Now, the total space of the principal $\mathrm{U}(1)$-bundle $\mathcal{L}$ of our model is the set of equivalence classes of above pairs:

$$
\mathcal{L}:=\{(\phi, z)\} / \sim .
$$

The bundle projection sends $(\phi, z)$ to $\partial \phi \in L G$, and the $\mathrm{U}(1)$-action is given by multiplication in the $\mathrm{U}(1)$-component. One can show that

$$
\mathrm{c}_{1}(\mathcal{L})=-\tau\left(\gamma_{\text {can }}\right) .
$$

This is already enough to identify the universal central extension.

The group structure on $\mathcal{L}$ turning it into a central extension is given by the Mickelsson product [11],

$$
\mathcal{L} \times \mathcal{L} \rightarrow \mathcal{L}, \quad\left(\left(\phi_{1}, z_{1}\right),\left(\phi_{2}, z_{2}\right)\right) \mapsto\left(\phi_{1} \phi_{2}, z_{1} z_{2} \cdot \exp \left(-2 \pi \mathrm{i} \int_{D^{2}}\left(\phi_{1}, \phi_{2}\right)^{*} \rho\right)\right),
$$

where $\rho$ is defined by

$$
\rho:=\frac{1}{2}\left\langle\operatorname{pr}_{1}^{*} \theta \wedge \operatorname{pr}_{2}^{*} \bar{\theta}\right\rangle \in \Omega^{2}(G \times G) .
$$

The two differential forms $H$ and $\rho$, which are the only parameters of the construction, satisfy the identities

$$
H_{g_{1} g_{2}}=H_{g_{1}}+H_{g_{2}}-\mathrm{d} \rho_{g_{1}, g_{2}} \quad \text { and } \quad \rho_{g_{1}, g_{2}}+\rho_{g_{1} g_{2}, g_{3}}=\rho_{g_{2}, g_{3}}+\rho_{g_{1}, g_{2} g_{3}}
$$

for all $g_{1}, g_{2}, g_{3} \in G$. The first identity assures the well-definedness of the Mickelsson product on equivalence classes, and the second implies its associativity.

Now we come to the fusion product. For $\left(\gamma_{1}, \gamma_{2}, \gamma_{3}\right) \in P G^{[3]}$, we define

$$
\begin{aligned}
\lambda_{\gamma_{1}, \gamma_{2}, \gamma_{3}}: \mathcal{L}_{\gamma_{1} \cup \gamma_{2}} \otimes \mathcal{L}_{\gamma_{2} \cup \gamma_{3}} & \rightarrow \mathcal{L}_{\gamma_{1} \cup \gamma_{3}}, \\
\left(\phi_{12}, z_{12}\right) \otimes\left(\phi_{23}, z_{23}\right) & \mapsto\left(\phi_{13}, z_{12} z_{23} \cdot \mathrm{e}^{2 \pi \mathrm{i} S_{\mathrm{WZ}}(\Psi)}\right),
\end{aligned}
$$


where $\phi_{13}: D^{2} \rightarrow G$ is an arbitrarily chosen smooth map with $\partial \phi_{13}=\gamma_{1} \cup \gamma_{3}$, and $\Psi: S^{2} \rightarrow G$ is obtained by trisecting $S^{2}$ along the longitudes $0,2 \pi / 3$ and $4 \pi / 3$, and prescribing $\Psi$ on each sector with the maps $\phi_{12}, \phi_{23}$ (with orientation-reversing identification) and $\phi_{13}$ (with orientation-preserving identification). This map $\Psi$ is smooth due to the sitting instants of the paths and the requirement that the maps $\phi_{i j}$ are radially constant.

That definition (7) of the fusion product on $\mathcal{L}$ is independent of the choice of $\phi_{13}$ follows from the identity

$$
S_{\mathrm{WZ}}(\Psi)=S_{\mathrm{WZ}}\left(\Psi^{\prime}\right) S_{\mathrm{WZ}}\left(\Phi_{13}\right)
$$

for Wess-Zumino terms, where $\Psi^{\prime}$ is obtained as described above, but using a different map $\phi_{13}^{\prime}$ instead of $\phi_{13}$, and $\Phi_{13}$ is obtained in the way described earlier from $\phi_{13}$ and $\phi_{13}^{\prime}$. Definition (7) is also well-defined under the equivalence relation $\sim$ due to a similar identity for Wess-Zumino terms. Finally, the fusion product on $\mathcal{L}$ is associative in the sense of Definition 3.3.

Now that we have explained that $\overline{L \operatorname{Spin}(n)}$ is a fusion extension, we proceed to introduce our new version of a spin structure on $L M$.

Definition 3.6 A fusion spin structure on $L M$ is a spin structure $(\mathcal{S}, \sigma)$ together with a fusion product $\lambda_{\mathcal{S}}$ on the associated principal $\mathrm{U}(1)$-bundle $\sigma: \mathcal{S} \rightarrow L F M$ of Lemma 3.2, such that the $\widetilde{L \operatorname{Spin}(n)}$-action on $\mathcal{S}$ is fusion-preserving,

$$
\lambda_{S}\left(q_{12} \cdot \beta_{12} \otimes q_{23} \cdot \beta_{23}\right)=\lambda_{S}\left(q_{12} \otimes q_{23}\right) \cdot \lambda\left(\beta_{12} \otimes \beta_{23}\right),
$$

where $\lambda$ is the fusion product of the fusion extension $\widetilde{L \operatorname{Spin}(n)}$, and the elements $q_{12}, q_{23} \in \mathcal{S}$ and $\beta_{12}, \beta_{23} \in \widehat{L \operatorname{Spin}(n)}$ are supposed to be such that the fusion products are defined.

Explicitly, the condition on the elements means there are paths $\left(\alpha_{1}, \alpha_{2}, \alpha_{3}\right) \in P F M^{[3]}$ and $\left(\gamma_{1}, \gamma_{2}, \gamma_{3}\right) \in P \operatorname{Spin}(n)^{[3]}$ such that $\sigma\left(q_{i j}\right)=\alpha_{i} \cup \alpha_{j}$ and $p\left(\beta_{i j}\right)=\gamma_{i} \cup \gamma_{j}$.

\section{Lifting gerbes for fusion extensions}

The main objective of this section is to embed the definition of a fusion spin structure into a more general theory. The results we derive in this setting will be used in the proof of the main result. 


\subsection{Lifting gerbes}

We briefly review the theory of lifting bundle gerbes for the convenience of the reader, following [12, Section 4]. The setup is a central extension

$$
1 \longrightarrow A \longrightarrow \widehat{G} \stackrel{t}{\longrightarrow} G \longrightarrow 1
$$

of (possibly Fréchet) Lie groups, and a principal $G$-bundle $P$ over a (possibly Fréchet) manifold $X$.

A $\widehat{G}$-lift of $P$ is a principal $\widehat{G}$-bundle $\widehat{P}$ over $X$ together with a bundle map $f: \widehat{P} \rightarrow P$ satisfying $f(\widehat{p} \cdot \widehat{g})=f(\widehat{p}) \cdot t(\widehat{g})$ for all $\widehat{p} \in \widehat{P}$ and $\widehat{g} \in \widehat{G}$. The existence of $\widehat{G}$-lifts is obstructed by a class $\xi_{P} \in \check{\mathrm{H}}^{2}(X, \underline{A})$ obtained in the following way. Suppose the principal $G$-bundle $P$ has transition functions $g_{\alpha \beta}: U_{\alpha} \cap U_{\beta} \rightarrow G$ with respect to an open cover of $X$. Then $\xi_{P}$ is the image of the Čech cohomology class of $g_{\alpha \beta}$ under the connecting homomorphism

$$
\check{\mathrm{H}}^{1}(X, \underline{G}) \rightarrow \check{\mathrm{H}}^{2}(X, \underline{A}) .
$$

Explicitly, upon passing to an appropriate refinement, one chooses lifts $\widehat{g}_{\alpha \beta}: U_{\alpha} \cap U_{\beta} \rightarrow$ $\widehat{G}$. These satisfy the cocycle condition up to a Čech 2-cochain $f_{\alpha \beta \gamma}: U_{\alpha} \cap U_{\beta} \cap U_{\gamma} \rightarrow$ $A$, and $\xi_{P}$ is the cohomology class of $f_{\alpha \beta \gamma}$. The class $\xi_{P}$ vanishes if and only if one can choose lifts $\widehat{g}_{\alpha \beta}$ that actually form a Čech cocycle. This, in turn, is equivalent to the existence of a $\widehat{G}$-lift $\widehat{P}$.

A stronger statement (Theorem 4.2) can be achieved about the category $\widehat{G}-\mathcal{L}$ ift $(P)$ of $\widehat{G}$-lifts of the principal $G$-bundle $P$. For this purpose we consider the lifting bundle gerbe $\mathcal{G}_{P}$ over $X$ associated to $P$. Its surjective submersion is the bundle projection $\pi: P \rightarrow X$. Over the two-fold fiber product $P^{[2]}:=P \times_{X} P$, the lifting bundle gerbe has the principal $A$-bundle $Q:=\delta^{*} \widehat{G}$, obtained by regarding $\widehat{G}$ as a principal $A$-bundle over $G$, and pulling it back along the "difference map"

$$
\delta: P^{[2]} \rightarrow G, \quad \text { with } p \cdot \delta\left(p, p^{\prime}\right)=p^{\prime} .
$$

Finally, the multiplication of $\widehat{G}$ defines a bundle gerbe product, ie a bundle isomorphism

$$
\begin{aligned}
\mu: \operatorname{pr}_{12}^{*} Q \otimes \operatorname{pr}_{23}^{*} Q & \rightarrow \operatorname{pr}_{13}^{*} Q, \\
\left(p_{1}, p_{2}, g_{12}\right) \otimes\left(p_{2}, p_{3}, g_{23}\right) & \mapsto\left(p_{1}, p_{3}, g_{12} g_{23}\right)
\end{aligned}
$$

over $P^{[3]}$ that is associative over $P^{[4]}$. Here, $\operatorname{pr}_{i j}: P^{[3]} \rightarrow P^{[2]}$ denote the projections to the indexed components. The characteristic class of the lifting gerbe $\mathcal{G}_{P}$ in $\check{\mathrm{H}}^{2}(X, \underline{A})$ coincides with the obstruction class $\xi_{P}$. In the case of $A=\mathrm{U}(1)$, it can be identified with a class in $\mathrm{H}^{3}(X, \mathbb{Z})$, the Dixmier-Douady class of $\mathcal{G}_{P}$. 
Example 4.1 If $P=F M$ is the oriented frame bundle of an oriented Riemannian manifold, and the central extension is

$$
1 \rightarrow \mathbb{Z}_{2} \rightarrow \operatorname{Spin}(n) \rightarrow \mathrm{SO}(n) \rightarrow 1,
$$

the associated lifting gerbe $\mathcal{G}_{F M}$ is the spin lifting gerbe of $M$. Its characteristic class $\xi_{F M} \in \check{\mathrm{H}}^{2}\left(M, \mathbb{Z}_{2}\right)$ is the second Stiefel-Whitney class $w_{2}$.

A trivialization of a bundle gerbe $\mathcal{G}$ is an isomorphism $\mathcal{T}: \mathcal{G} \rightarrow \mathcal{I}$, where $\mathcal{I}$ denotes the trivial bundle gerbe [21]. Trivializations form a category we denote by $\operatorname{Triv}(\mathcal{G})$. In case of the lifting bundle gerbe $\mathcal{G}_{P}$, a trivialization is a principal $A$-bundle $T$ over $P$ together with a bundle isomorphism

$$
\kappa: Q \otimes \operatorname{pr}_{2}^{*} T \rightarrow \operatorname{pr}_{1}^{*} T
$$

over $P^{[2]}$ satisfying a compatibility condition with the bundle gerbe product $\mu$, namely

$$
\kappa\left(q_{12} \otimes \kappa\left(q_{23} \otimes t\right)\right)=\kappa\left(\mu\left(q_{12} \otimes q_{23}\right) \otimes t\right)
$$

for all $\left(p_{1}, p_{2}, p_{3}\right) \in P^{[3]}$ and all $t \in T_{p_{3}}, q_{12} \in Q_{p_{1}, p_{2}}$, and $q_{23} \in Q_{p_{2}, p_{3}}$. We have the following result of [12, Section 4].

Theorem 4.2 Let $P$ be a principal $G$-bundle over $X$. Then the above constructions constitute an equivalence of categories,

$$
\operatorname{Triv}\left(\mathcal{G}_{P}\right) \cong \widehat{G}-\mathcal{L} \operatorname{ift}(P) .
$$

Let us briefly sketch the two functors that establish the equivalence of Theorem 4.2. Suppose $(T, \kappa)$ is a trivialization of $\mathcal{G}_{P}$. We equip $\widehat{P}:=T$ with the projection $T \rightarrow P \rightarrow X$ and the $\widehat{G}$-action $\widehat{p} \cdot \widehat{g}:=\kappa\left(\widehat{g}^{-1} \otimes \widehat{p}\right)$. This defines a principal $\widehat{G}-$ bundle over $X$. Together with the map $f: T \rightarrow P$ it forms a $\widehat{G}$-lift of $P$. Conversely, suppose $f: \widehat{P} \rightarrow P$ is a $\widehat{G}$-lift of $P$. We equip $T:=\widehat{P}$ with the map $f: T \rightarrow P$ and the $A$-action

$$
T \times A \rightarrow T, \quad(t, a) \mapsto t \cdot i\left(a^{-1}\right),
$$

where $i: A \rightarrow \widehat{G}$ is the inclusion of the central subgroup, and $\cdot$ is the principal $\widehat{G}$-action on $\widehat{P}$. This equips $T$ with the structure of a principal $A$-bundle over $P$. Together with the bundle morphism $\kappa$ defined by $\kappa(\widehat{g} \otimes \widehat{p}):=\widehat{p} \cdot \widehat{g}^{-1}$ it is a trivialization of $\mathcal{G}_{P}$.

Example 4.3 If $P=L F M$ is the looping of the frame bundle of a spin manifold, and $\widehat{G}=\widehat{L \operatorname{Spin}(n)}$ is the universal central extension of $G=L \operatorname{Spin}(n)$, then the spin structures on $L M$ of Section 3 form precisely the category $\widetilde{L \operatorname{Spin}(n)}-\mathcal{L} \operatorname{ift}(L F M)$. The obstruction class $\xi_{L F M} \in \mathrm{H}^{2}(L M, \underline{\mathrm{U}(1)})$ can be identified with a class in $\mathrm{H}^{3}(L M, \mathbb{Z})$; 
this is the spin class $\lambda_{L M}$ of $L M$ mentioned in Section 1. The lifting bundle gerbe is denoted $\mathcal{S}_{L M}$ and called the spin lifting gerbe. Its Dixmier-Douady class satisfies

$$
\operatorname{dd}\left(\mathcal{S}_{L M}\right)+\lambda_{L M}=0 .
$$

Theorem 4.2 implies an equivalence

$$
\{\text { Spin structures on } L M\} \cong\left\{\begin{array}{l}
\text { Trivializations of the } \\
\text { spin lifting gerbe } \mathcal{S}_{L M}
\end{array}\right\} .
$$

Under this equivalence, a spin structure $(\mathcal{S}, \sigma)$ corresponds to a trivialization $(T, \kappa)$ of $\mathcal{S}_{L M}$ whose principal $\mathrm{U}(1)$-bundle $T$ is the one of Lemma 3.2.

\subsection{Transgression and regression}

In this section, we explain the role of fusion products from a more general perspective. Based on Definition 3.3, fusion bundles with structure group $A$ over the loop space $L X$ of a smooth manifold $X$ form a category we denote by $\mathcal{F} u s \mathcal{B} u n_{A}(L X)$. These categories are monoidal and natural with respect to looped maps, ie if $f: X \rightarrow Y$ is a smooth map between smooth manifolds, and $L f: L X \rightarrow L Y$ denotes the induced map on loop spaces, pullback is a functor

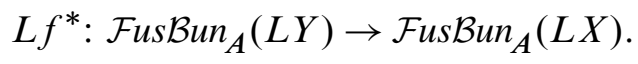

Fusion bundles are those bundles over $L X$ that correspond to bundle gerbes over $X$. This correspondence can be seen in both directions. First, fusion products furnish a regression functor

$$
\mathscr{R}_{X}: \mathcal{F u s B u n}_{A}(L X) \rightarrow \mathcal{G r b}_{A}(X),
$$

landing in the 2-category of bundle gerbes over $X$; see [26, Section 5.1]. The regression functor $\mathscr{R}_{X}$ is defined for connected manifolds $X$ and depends on the choice of a base point $x \in X$ (up to canonical, natural equivalence). It is weak (ie it has nontrivial compositor 2-morphisms), but induces a honest functor into the category $\mathrm{h}_{1} \mathcal{G r b}_{A}(X)$ obtained from the 2-category $\mathcal{G r b}_{A}(X)$ by identifying 2-isomorphic 1-morphisms. We remark two evident properties:

Lemma 4.4 The functor $\mathscr{R}_{x}$ has the following properties:

(i) It is monoidal.

(ii) It is natural with respect to smooth, base-point-preserving maps.

Second, in the other direction, there is a transgression functor

$$
\mathrm{h}_{1} \mathcal{G r b}{ }_{A}^{\nabla}(X) \rightarrow \mathcal{F} u s \mathcal{B} u n_{A}(L X), \quad \mathcal{G} \mapsto \mathscr{T}_{\mathcal{G}}
$$


defined on the category of $A$-bundle gerbes with connections over $X$. It was introduced by Brylinski and McLaughlin [5] and lifted to fusion bundles in [26, Section 4.2]. For $A=\mathrm{U}(1)$ it satisfies, on the level of characteristic classes, the relation

$$
\mathrm{c}_{1}\left(\mathscr{T}_{\mathcal{G}}\right)=-\tau(\operatorname{dd}(\mathcal{G})) \in \mathrm{H}^{2}(L X, \mathbb{Z}) .
$$

We shall describe some details of the transgression functor following [22; 26]. If $\mathcal{G}$ is a bundle gerbe with connection over $X$, the fiber of $\mathscr{T}_{\mathcal{G}}$ over a loop $\tau \in L X$ is

$$
\left.\mathscr{T}_{\mathcal{G}}\right|_{\tau}:=\mathrm{h}_{0} \operatorname{Triv}^{\nabla}\left(\tau^{*} \mathcal{G}\right)
$$

ie it consists of isomorphism classes of connection-preserving trivializations of $\tau^{*} \mathcal{G}$. A connection-preserving isomorphism $\mathcal{A}: \mathcal{G}_{1} \rightarrow \mathcal{G}_{2}$ induces a bundle morphism given by

$$
\mathscr{T}_{\mathcal{A}}: \mathscr{T}_{\mathcal{G}_{1}} \rightarrow \mathscr{T}_{\mathcal{G}_{2}}, \quad \mathcal{T} \mapsto \mathcal{T} \circ \tau^{*} \mathcal{A}^{-1}
$$

The lift of this construction to the category of fusion bundles over $L X$ is established by recognizing a fusion product $\lambda_{\mathcal{G}}$ on the bundle $\mathscr{T}_{\mathcal{G}}$. Let us recall how $\lambda_{\mathcal{G}}$ is characterized. We denote by $\iota_{1}, \iota_{2}:[0,1] \rightarrow S^{1}$ the inclusion of the interval into the left and the right half of the circle. Let $\left(\gamma_{1}, \gamma_{2}, \gamma_{3}\right)$ be a triple of paths with a common initial point $x$ and a common end point $y$, and let $\mathcal{T}_{i j}$ be trivializations of the pullback of $\mathcal{G}$ to the loops $\gamma_{i} \cup \gamma_{j}$, for $(i j)=(12),(23),(13)$. Then the relation

$$
\lambda_{\mathcal{G}}\left(\mathcal{T}_{12} \otimes \mathcal{T}_{23}\right)=\mathcal{T}_{13}
$$

holds if and only if there exist 2-isomorphisms

$$
\phi_{1}: \iota_{1}^{*} \mathcal{T}_{12} \Rightarrow \iota_{1}^{*} \mathcal{T}_{13}, \quad \phi_{2}: \iota_{2}^{*} \mathcal{T}_{12} \Rightarrow \iota_{1}^{*} \mathcal{T}_{23} \quad \text { and } \quad \phi_{3}: \iota_{2}^{*} \mathcal{T}_{23} \Rightarrow \iota_{2}^{*} \mathcal{T}_{13}
$$

between trivializations of the pullbacks of $\mathcal{G}$ to the paths $\gamma_{1}, \gamma_{2}$, and $\gamma_{3}$, respectively, whose restrictions to the two common points $x$ and $y$ satisfy the cocycle condition $\phi_{1}=\phi_{3} \circ \phi_{2}$.

Example 4.5 The orientation bundle $\mathcal{O}_{L M}$ of the loop space, mentioned in Section 2, is the transgression of the spin lifting gerbe $\mathcal{G}_{F M}$ of $M$; see Example 4.1. This explains the existence of a fusion product on $\mathcal{O}_{L M}$, independently of its discovery by Stolz and Teichner [20].

The regression functor $\mathscr{R}_{x}$ and the transgression functor $\mathscr{T}$ are inverse to each other, in a way that has various formulations. For the purpose of this article we need the following aspect. 
Theorem 4.6 The diagram

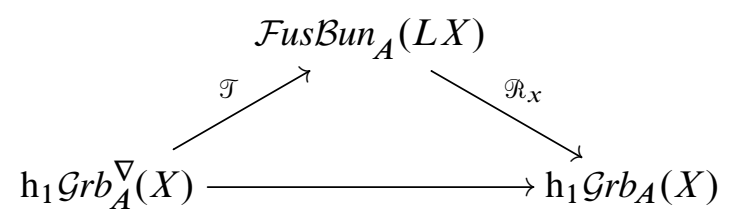

of functors whose bottom arrow is the functor that forgets connections, is commutative up to a canonical natural equivalence.

I remark that transgression and regression can be turned into an honest equivalence of categories, by either including the connection on the loop space side or dropping the connections on the gerbes; see the main results of $[26 ; 27]$.

\subsection{Multiplicative gerbes and fusion extensions}

In this section we explain how fusion extensions (Definition 3.4) can be obtained by transgression of multiplicative gerbes. Let $A$ be an abelian Lie group. We recall from [22, Definition 1.3] that a multiplicative A-bundle gerbe with connection over a Lie group $G$ is a triple $(\mathcal{G}, \rho, \mathcal{M}, \alpha)$ consisting of an $A$-bundle gerbe $\mathcal{G}$ with connection over $G$ together with a 2 -form $\rho \in \Omega^{2}(G \times G, \mathfrak{a})$ with values in the Lie algebra $\mathfrak{a}$ of $A$, a connection-preserving isomorphism

$$
\mathcal{M}: \operatorname{pr}_{1}^{*} \mathcal{G} \otimes \operatorname{pr}_{2}^{*} \mathcal{G} \rightarrow m^{*} \mathcal{G} \otimes \mathcal{I}_{\rho}
$$

between gerbes over $G \times G$, and a connection-preserving transformation $\alpha$ over $G \times G \times G$, that serves as an associator for the multiplication (20) and satisfies the pentagon axiom. In (20) we have denoted by $\mathcal{I}_{\rho}$ the trivial bundle gerbe equipped with the curving 2-form $\rho$. That (20) is connection-preserving implies for $H:=\operatorname{curv}(\mathcal{G}) \in$ $\Omega^{3}(G)$ the identity

$$
H_{g_{1} g_{2}}=H_{g_{1}}+H_{g_{2}}-\mathrm{d} \rho_{g_{1}, g_{2}}
$$

and the existence of $\alpha$ implies the identity

$$
\rho_{g_{1}, g_{2}}+\rho_{g_{1} g_{2}, g_{3}}=\rho_{g_{2}, g_{3}}+\rho_{g_{1}, g_{2} g_{3}} .
$$

Let us first explain how a central extension is produced from a multiplicative bundle gerbe with connection. The transgression of $\mathcal{G}$ is a principal $A$-bundle $\mathcal{G}:=\mathscr{T}_{\mathcal{G}}$ over $L G$. Next, the transgression of $\mathcal{M}$ is a bundle isomorphism

$$
\mathcal{T}_{\mathcal{M}}: \operatorname{pr}_{1}^{*} \mathcal{G} \otimes \operatorname{pr}_{2}^{*} \mathcal{G} \rightarrow m^{*} \mathcal{G} \otimes \mathscr{T}_{\mathcal{I}_{\rho}}
$$


As described in [22, Section 3.1] the U(1)-bundle $\mathscr{T}_{\mathcal{I}_{\rho}}$ has a canonical trivialization

$$
t_{\rho}: \mathscr{T}_{\mathcal{I}_{\rho}} \rightarrow \mathbf{I}
$$

Together, we obtain a bundle isomorphism

$$
\operatorname{pr}_{1}^{*} \mathcal{G} \otimes \operatorname{pr}_{2}^{*} \mathcal{G} \stackrel{\mathcal{T}_{\mathcal{M}}}{\longrightarrow} m^{*} \mathcal{G} \otimes \mathcal{I}_{\mathcal{I}_{\rho}} \stackrel{\operatorname{id} \otimes t_{\rho}}{\longrightarrow} m^{*} \mathcal{G} .
$$

This bundle morphism induces a smooth map $\tilde{m}: \mathcal{G} \times \mathcal{G} \rightarrow \mathcal{G}$ covering the multiplication of $L G$ along the projection $\mathcal{G} \rightarrow L G$. The transgression of the associator $\alpha$ guarantees the associativity of $\tilde{m}$. Then the principal $A$-bundle $\mathcal{G}$ together with this product becomes a central extension

$$
1 \rightarrow A \rightarrow \mathcal{G} \rightarrow L G \rightarrow 1
$$

of Fréchet Lie groups [22, Theorem 3.1.7].

Theorem 4.7 Let $\mathcal{G}$ be a multiplicative $A$-bundle gerbe with connection over $G$. Then $\mathscr{T}_{\mathcal{G}}$ is a fusion extension of $L G$ by $A$.

Proof We notice that $\mathscr{T}_{\mathcal{G}}$ is a fusion bundle; see (15). The bundle morphism (24) is fusion-preserving, since the isomorphism $\mathcal{M}$ transgresses to a fusion-preserving bundle morphism, and the trivialization (23) is fusion-preserving [23, Lemma 3.6]. This shows the multiplicativity of the fusion product of $\mathscr{T}_{\mathcal{G}}$ in the sense of Definition 3.4.

Suppose that $G$ is a compact, connected, simple and simply connected Lie group. Let $\gamma_{\text {can }} \in \mathrm{H}^{3}(G, \mathbb{Z})$ be a generator. There is a canonical multiplicative $\mathrm{U}(1)$-bundle gerbe with connection, the basic bundle gerbe $\mathcal{G}_{\text {bas }}$, whose Dixmier-Douady class is $\gamma_{\text {can }}$; see [22, Example 1.5] and [24]. The curvature of $\mathcal{G}_{\text {bas }}$ is $H=\frac{1}{6}\langle\theta \wedge[\theta \wedge \theta]\rangle$, and the 2 -form of its multiplicative structure is $\rho=\frac{1}{2}\left\langle\operatorname{pr}_{1}^{*} \theta \wedge \operatorname{pr}_{2}^{*} \bar{\theta}\right\rangle$; both forms appeared already in Section 3. Since

$$
\mathrm{c}_{1}\left(\mathscr{T}_{\mathcal{G}_{\text {bas }}}\right) \stackrel{(16)}{=}-\tau\left(\operatorname{dd}\left(\mathcal{G}_{\text {bas }}\right)\right)=-\tau\left(\gamma_{\text {can }}\right)
$$

we have, according to our sign convention (3), the following consequence.

Corollary 4.8 Suppose that $G$ is a compact, connected, simple and simply connected Lie group. Then $\mathscr{T}_{\mathcal{G}}$ bas is the universal central extension $\widetilde{L G}$. In particular, the universal central extension of $L G$ is a fusion extension.

We have now described two models for the universal central extension of a compact, connected, simple and simply connected Lie group: the explicit construction $\mathcal{L}$ of Section 3 , and the transgression $\mathscr{T}_{\mathcal{G}_{\text {bas }}}$ of the basic bundle gerbe. Both models are fusion 
extensions, ie they come equipped with multiplicative fusion products. In the remainder of this section we show that the two models are isomorphic as fusion extensions.

We shall first describe an explicit isomorphism between the underlying principal U(1)bundles of the two models. It is given by the formula

$$
\varphi: \mathcal{L} \rightarrow \mathscr{T}_{\mathcal{G}_{\text {bas }}}, \quad(\phi, z) \mapsto \partial \mathcal{T} \cdot z \cdot \exp \left(-2 \pi \mathrm{i} \int_{D^{2}} \omega\right)
$$

Here $\mathcal{T}: \phi^{*} \mathcal{G}_{\text {bas }} \rightarrow \mathcal{I}_{\omega}$ is an arbitrarily chosen trivialization of $\phi^{*} \mathcal{G}_{\text {bas }}$ over $D^{2}$ and $\partial \mathcal{T}$ denotes its restriction to the boundary; the latter is a trivialization of $\partial \phi^{*} \mathcal{G}_{\text {bas }}$ over $S^{1}$, ie an element in $\mathscr{T}_{\mathcal{G}_{\text {bas }}}$ over the loop $\partial \phi$; see (17).

Proposition 4.9 The formula (25) defines an isomorphism of principal U(1)-bundles over $L G$.

Proof The main point is to show the independence of $\varphi$ from the choice of $\mathcal{T}$ and its well-definedness under the equivalence relation from the definition of $\mathcal{L}$. If $\mathcal{T}^{\prime}: \phi^{*} \mathcal{G}_{\text {bas }} \rightarrow \mathcal{I}_{\omega^{\prime}}$ is a different choice of a trivialization, then there exists a principal $\mathrm{U}(1)$-bundle $P$ with connection over $D^{2}$ with $\operatorname{curv}(P)=\omega^{\prime}-\omega$ and $\partial \mathcal{T}^{\prime}=\partial \mathcal{T}$. $\operatorname{Hol}_{P}\left(S^{1}\right)$ as elements in $\left.\mathscr{T}_{\mathcal{G}_{\text {bas }}}\right|_{\partial \phi}$; see [22, Section 3.1] for some more details. Since

$$
\operatorname{Hol}_{P}\left(\partial D^{2}\right)=\exp \left(2 \pi \mathrm{i} \int_{D^{2}} \operatorname{curv}(P)\right)
$$

the two contributions cancel. Now suppose that $\left(\phi^{\prime}, z^{\prime}\right)$ is an equivalent representative of the element in $\mathcal{L}$, ie $\partial \phi=\partial \phi^{\prime}$ and $z=z^{\prime} \cdot \mathrm{e}^{2 \pi \mathrm{i} S_{\mathrm{WZ}}(\Phi)}$. We may choose a trivialization $\widetilde{\mathcal{T}}: \Phi^{*} \mathcal{G}_{\text {bas }} \rightarrow \mathcal{I}_{\widetilde{\omega}}$ and use its restrictions $\mathcal{T}$ and $\mathcal{T}^{\prime}$ to the two hemispheres in the definition of $\varphi$. Now, well-definedness follows from the fact that

$$
\exp \left(2 \pi \mathrm{i} S_{\mathrm{WZ}}(\Phi)\right)=\exp \left(2 \pi \mathrm{i} \int_{D^{3}} \tilde{\Phi}^{*} H\right)=\exp \left(2 \pi \mathrm{i} \int_{S^{2}} \tilde{\omega}\right),
$$

the latter equality being a consequence of the integrality of $H$ and the relation $\Phi^{*} H=$ $\operatorname{curv}\left(\Phi^{*} \mathcal{G}_{\text {bas }}\right)=\mathrm{d} \widetilde{\omega}$ (as for any trivialization). Finally, the map $\varphi$ is fiber-preserving and $\mathrm{U}(1)$-equivariant; hence, a bundle isomorphism.

Proposition 4.10 The bundle isomorphism $\varphi$ is a group homomorphism and fusionpreserving, and thus defines an equivalence between the fusion extensions $\mathscr{T}_{\mathcal{G}_{\text {bas }}}$ and $\mathcal{L}$.

Proof In order to see that $\varphi$ preserves the group structure, consider two elements $\left(\phi_{1}, z_{1}\right)$ and $\left(\phi_{2}, z_{2}\right)$ in $\mathcal{L}$, and recall that

$$
\left(\phi_{1}, z_{1}\right) \cdot\left(\phi_{2}, z_{2}\right)=\left(\phi_{1} \phi_{2}, z_{1} z_{2} \cdot \exp \left(-2 \pi \mathrm{i} \int_{D^{2}}\left(\phi_{1}, \phi_{2}\right)^{*} \rho\right)\right) \text {. }
$$


Let $\mathcal{T}_{1}: \phi_{1}^{*} \mathcal{G}_{\text {bas }} \rightarrow \mathcal{I}_{\omega_{1}}$ and $\mathcal{T}_{2}: \phi_{2}^{*} \mathcal{G}_{\text {bas }} \rightarrow \mathcal{I}_{\omega_{2}}$ be trivializations. We define a new trivialization $\mathcal{T}_{12}:\left(\phi_{1} \phi_{2}\right)^{*} \mathcal{G}_{\text {bas }} \rightarrow \mathcal{I}_{\omega_{12}}$ as the composite

$\left(\phi_{1} \phi_{2}\right)^{*} \mathcal{G}_{\mathrm{bas}}=\left(\phi_{1}, \phi_{2}\right)^{*} m^{*} \mathcal{G}_{\mathrm{bas}} \otimes \mathcal{I}_{\left(\phi_{1}, \phi_{2}\right)^{*} \rho} \otimes \mathcal{I}_{-\left(\phi_{1}, \phi_{2}\right)^{*} \rho}$

$$
\phi_{1}^{*} \mathcal{G}_{\text {bas }} \otimes \phi_{2}^{*} \mathcal{G}_{\text {bas }}^{\downarrow} \otimes \mathcal{I}_{-\left(\phi_{1}, \phi_{2}\right)^{*} \rho} \stackrel{ }{\mathcal{T}_{1} \otimes \mathcal{T}_{2} \otimes \mathrm{id}} \longrightarrow \mathcal{I}_{\omega_{1}+\omega_{2}-\left(\phi_{1}, \phi_{2}\right)^{*} \rho}
$$

Restricting to the boundary $S^{1}=\partial D^{2}$, we have $\partial \mathcal{T}_{12}=\partial \mathcal{T}_{1} \cdot \partial \mathcal{T}_{2}$ in the group structure of the Fréchet Lie group $\mathscr{T}_{\mathcal{G}_{\text {bas }}}$, compare (18) and (24). Hence

$$
\begin{aligned}
\varphi\left(\phi_{1}, z_{1}\right) \cdot \varphi\left(\phi_{2}, z_{2}\right) & =\partial \mathcal{T}_{1} \cdot \partial \mathcal{T}_{2} \cdot z_{1} z_{2} \cdot \exp \left(-2 \pi \mathrm{i} \int_{D^{2}} \omega_{1}+\omega_{2}\right) \\
& =\partial \mathcal{T}_{12} \cdot z_{1} z_{2} \cdot \exp \left(-2 \pi \mathrm{i} \int_{D^{2}} \omega_{12}+\left(\phi_{1}, \phi_{2}\right)^{*} \rho\right) \\
& =\varphi\left(\phi_{1} \phi_{2}, z_{1} z_{2}\right) \cdot \exp \left(-2 \pi \mathrm{i} \int_{D^{2}}\left(\phi_{1}, \phi_{2}\right)^{*} \rho\right) \\
& =\varphi\left(\left(\phi_{1}, z_{1}\right) \cdot\left(\phi_{2}, z_{2}\right)\right) .
\end{aligned}
$$

This shows that $\varphi$ is a group homomorphism.

In order to see that $\varphi$ is fusion-preserving, we assume that $\left(\gamma_{1}, \gamma_{2}, \gamma_{3}\right) \in P G^{[3]}$, that $\phi_{i j}: D^{2} \rightarrow G$ are smooth maps with $\partial \phi_{i j}=\gamma_{i} \cup \gamma_{j}$, and that $\Psi: S^{2} \rightarrow G$ is constructed from the latter ones as described in Section 3. Thus, we have

$$
\lambda_{\mathcal{L}}\left(\left(\phi_{12}, z_{12}\right) \otimes\left(\phi_{23}, z_{23}\right)\right)=\left(\phi_{13}, z_{12} z_{23} \cdot \mathrm{e}^{2 \pi \mathrm{i} S_{\mathrm{WZ}}(\Psi)}\right) .
$$

We choose a trivialization $\mathcal{T}: \Psi^{*} \mathcal{G}_{\text {bas }} \rightarrow \mathcal{I}_{\omega}$, and restrict to trivializations $\mathcal{T}_{i j}: \phi_{i j}^{*} \mathcal{G}_{\text {bas }} \rightarrow$ $\mathcal{I}_{\omega_{i j}}$. In this situation, Definition (19) of the fusion product $\lambda_{\mathcal{G}_{\text {bas }}}$ on $\mathscr{T}_{\mathcal{G}_{\text {bas }}}$ shows that

$$
\lambda_{\mathcal{G}_{\text {bas }}}\left(\partial \mathcal{T}_{12} \otimes \partial \mathcal{T}_{23}\right)=\partial \mathcal{T}_{13}
$$

Thus

$$
\begin{aligned}
\lambda_{\mathcal{G}_{\text {bas }}}\left(\varphi\left(\phi_{12}, z_{12}\right) \otimes \varphi\left(\phi_{23}, z_{23}\right)\right) & =\partial \mathcal{T}_{13} \cdot z_{12} z_{23} \cdot \exp \left(-2 \pi \mathrm{i} \int_{D^{2}}\left(\omega_{12}+\omega_{23}\right)\right) \\
& =\partial \mathcal{T}_{13} \cdot z_{12} z_{23} \cdot \exp \left(-2 \pi \mathrm{i} \int_{D^{2}}\left(\omega_{13}-\omega\right)\right) \\
& =\partial \mathcal{T}_{13} \cdot z_{12} z_{23} \cdot \mathrm{e}^{2 \pi \mathrm{i} S_{\mathrm{WZ}}(\Psi)} \cdot \exp \left(-2 \pi \mathrm{i} \int_{D^{2}} \omega_{13}\right) \\
& =\varphi\left(\phi_{13}, z_{12} z_{23} \cdot \mathrm{e}^{2 \pi \mathrm{i} S_{\mathrm{WZ}}(\Psi)}\right) .
\end{aligned}
$$

This shows that $\varphi$ is fusion-preserving. 


\subsection{Fusion lifts}

In Section 4.1 we have obtained a formulation of spin structures on $L M$ in terms of trivializations of the spin lifting gerbe $\mathcal{S}_{L M}$. The goal of this section is to extend this formulation to fusion spin structures of Definition 3.6 (see Corollary 4.17 below).

For this purpose we develop a general theory for lifting problems along fusion extensions. We show that lifting gerbes for fusion extensions are equipped with certain additional structure that we call internal fusion product (Definition 4.13). The relevant trivializations are then identified as those that respect this internal fusion product (Theorem 4.16).

We consider a connected Lie group $G$, an abelian Lie group $A$, a fusion extension

$$
1 \rightarrow A \rightarrow \mathcal{G} \rightarrow L G \rightarrow 1,
$$

and a principal $G$-bundle $E$ over a smooth manifold $M$. The fusion product on $\mathcal{G}$ will be denoted by $\lambda_{g}$. We recall that $L E$ is a Fréchet principal $L G$-bundle over $L M$.

Definition 4.11 A fusion lift of the structure group of $L E$ from $L G$ to $\mathcal{G}$ is a $\mathcal{G}-$ lift $(\mathcal{S}, \sigma)$ together with a fusion product $\lambda_{\mathcal{S}}$ on the associated principal $A$-bundle $\sigma: \mathcal{S} \rightarrow L E$, such that the $\mathcal{G}$-action on $\mathcal{S}$ is fusion-preserving,

$$
\lambda_{\varepsilon}\left(q_{12} \cdot \beta_{12} \otimes q_{23} \cdot \beta_{23}\right)=\lambda_{\varepsilon}\left(q_{12} \otimes q_{23}\right) \cdot \lambda_{\mathcal{G}}\left(\beta_{12} \otimes \beta_{23}\right)
$$

for all $q_{i j} \in \mathcal{S}_{\alpha_{i} \cup \alpha_{j}}, \beta_{i j} \in \mathcal{G}_{\gamma_{i} \cup \gamma_{j}}$, and all $\left(\alpha_{1}, \alpha_{2}, \alpha_{3}\right) \in P E^{[3]}$ and $\left(\gamma_{1}, \gamma_{2}, \gamma_{3}\right) \in$ $P G^{[3]}$.

Here, by associated A-bundle we mean the bundle defined analogously to the one of Lemma 3.2: its total space is $\mathcal{S}$ and its bundle projection is the map $\sigma: \mathcal{S} \rightarrow L E$. The principal $A$-action is given by the formula

$$
\mathcal{S} \times A \rightarrow \mathcal{S}, \quad(q, a) \mapsto \mathcal{S} \cdot i\left(a^{-1}\right),
$$

where $i: A \rightarrow \mathcal{G}$ is the inclusion of the central subgroup, and $\cdot$ is the principal $\mathcal{G}-$ action on $\mathcal{S}$. A morphism between fusion lifts $\left(\mathcal{S}_{1}, \sigma_{1}, \lambda_{\mathcal{S}_{1}}\right)$ and $\left(\mathcal{S}_{2}, \sigma_{2}, \lambda_{\mathcal{S}_{2}}\right)$ is called fusion-preserving, if the induced morphism $\mathcal{S}_{1} \rightarrow \mathcal{S}_{2}$ of principal $A$-bundles over $L E$ preserves the fusion products $\lambda_{1}$ and $\lambda_{2}$. Fusion lifts form a category denoted by $\mathcal{G}-\mathcal{F} u s \mathcal{L}$ ift $(L E)$.

Example 4.12 In this notation, the fusion spin structures introduced in Definition 3.6 form precisely the category $\widetilde{L \operatorname{Spin}(n)}-\mathcal{F} u S \mathcal{L}$ ift $(L F M)$. 
In the following we describe fusion lifts in terms of lifting bundle gerbes, and equip, for this purpose, the ordinary lifting bundle gerbe $\mathcal{G}_{L E}$ for lifting the looped bundle $L E$ from $L G$ to $\mathcal{G}$ with an additional structure.

Definition 4.13 Let $\mathcal{G}$ be an $A$-bundle gerbe over $L M$ whose surjective submersion is the looping of a surjective submersion $\pi: Y \rightarrow M$. Let $P$ denote its principal $A$-bundle over $L Y^{[2]}$ and $\mu$ denote its bundle gerbe product. An internal fusion product on $\mathcal{G}$ is a fusion product on $P$ such that $\mu$ is fusion-preserving.

The condition that $\mu$ is fusion-preserving makes sense since $\mu$ is a morphism

$$
\mu: \operatorname{pr}_{12}^{*} P \otimes \operatorname{pr}_{23}^{*} P \rightarrow \operatorname{pr}_{13}^{*} P
$$

between fusion bundles over $L Y^{[3]}$. In order to spell it out explicitly, we must consider three paths $\gamma_{1}, \gamma_{2}, \gamma_{3} \in P Y^{[3]}$ with a common initial point in $Y^{[3]}$ and a common end point in $Y^{[3]}$. The composition of a path $\gamma_{k}$ with one of the projections $\operatorname{pr}_{i}: Y^{[3]} \rightarrow P Y$ gives a path $\gamma_{k}^{i} \in P Y$, and these give in turn loops which we denote by $\tau_{i j}:=\gamma_{i}^{1} \cup \gamma_{j}^{1} \in$ $L Y$ and $\tau_{i j}^{\prime}=\gamma_{i}^{2} \cup \gamma_{j}^{2} \in L Y$, as well as $\tau_{i j}^{\prime \prime}=\gamma_{i}^{3} \cup \gamma_{j}^{3} \in L Y$. Now, the condition is that

$$
\mu\left(\lambda\left(q_{12} \otimes q_{23}\right) \otimes \lambda\left(q_{12}^{\prime} \otimes q_{23}^{\prime}\right)\right)=\lambda\left(\mu\left(q_{12} \otimes q_{12}^{\prime}\right) \otimes \mu\left(q_{23} \otimes q_{23}^{\prime}\right)\right)
$$

for all $q_{i j} \in P_{\left(\tau_{i j}, \tau_{i j}^{\prime}\right)}$ and $q_{i j}^{\prime} \in P_{\left(\tau_{i j}^{\prime}, \tau_{i j}^{\prime \prime}\right)}$.

There are two important examples of bundle gerbes with internal fusion products. One is when the bundle gerbe $\mathcal{G}$ is the transgression of a bundle 2 -gerbe over $M$; this will be explained in Section 5.1. The other example is when $\mathcal{G}$ is the lifting bundle gerbe $\mathcal{G}_{L E}$ associated to the problem of lifting the structure group of $L E$ from $L G$ to the fusion extension $\mathcal{G}$.

Indeed, in this case the surjective submersion of $\mathcal{G}_{L E}$ is the looping of the bundle projection $E \rightarrow M$, and the principal $A$-bundle $P=L \delta^{*} \mathcal{G}$ is the pullback of a fusion bundle along a looped map, and thus a fusion bundle; see (13). We will denote this internal fusion product on $\mathcal{G}_{L E}$ by $\lambda_{L E}$.

In order to check that the bundle gerbe product (10) is fusion-preserving, we consider paths $\gamma_{1}, \gamma_{2}, \gamma_{3} \in P E^{[3]}$ as above, and write $q_{i j}=\left(\tau_{i j}, \tau_{i j}^{\prime}, \beta_{i j}\right)$ and $q_{i j}^{\prime}=$ $\left(\tau_{i j}^{\prime}, \tau_{i j}^{\prime \prime}, \beta_{i j}^{\prime}\right)$ for elements $\beta_{i j} \in \mathcal{G}_{\gamma_{i j}}$ and $\beta_{i j}^{\prime} \in \mathcal{G}_{\gamma_{i j}^{\prime}}$, where $\gamma_{i j}:=L \delta\left(\tau_{i j}, \tau_{i j}^{\prime}\right) \in L G$ and $\gamma_{i j}^{\prime}:=L \delta\left(\tau_{i j}^{\prime}, \tau_{i j}^{\prime \prime}\right) \in L G$. Note that now

$$
\begin{aligned}
\lambda_{L E}\left(q_{12} \otimes q_{23}\right) & =\lambda_{L E}\left(\left(\tau_{12}, \tau_{12}^{\prime}, \beta_{12}\right) \otimes\left(\tau_{23}, \tau_{23}^{\prime}, \beta_{23}\right)\right) \\
& =\left(\tau_{13}, \tau_{13}^{\prime}, \lambda_{\mathcal{G}}\left(\beta_{12} \otimes \beta_{23}\right)\right),
\end{aligned}
$$


and similarly for the primed elements. Then we calculate $\mu\left(\lambda_{L E}\left(q_{12} \otimes q_{23}\right) \otimes \lambda_{L E}\left(q_{12}^{\prime} \otimes q_{23}^{\prime}\right)\right)$

$$
\begin{aligned}
& \stackrel{(26)}{=} \mu\left(\left(\tau_{13}, \tau_{13}^{\prime}, \lambda_{\mathcal{G}}\left(\beta_{12} \otimes \beta_{23}\right)\right) \otimes\left(\tau_{13}^{\prime}, \tau_{13}^{\prime \prime}, \lambda_{\mathcal{G}}\left(\beta_{12}^{\prime} \otimes \beta_{23}^{\prime}\right)\right)\right) \\
& \stackrel{(10)}{=}\left(\tau_{13}, \tau_{13}^{\prime \prime}, \lambda_{\mathcal{G}}\left(\beta_{12}^{\prime} \otimes \beta_{23}^{\prime}\right) \cdot \lambda_{\mathcal{G}}\left(\beta_{12} \otimes \beta_{23}\right)\right) \\
& \stackrel{(4)}{=}\left(\tau_{13}, \tau_{13}^{\prime \prime}, \lambda_{\mathcal{G}}\left(\beta_{12}^{\prime} \beta_{12} \otimes \beta_{23}^{\prime} \beta_{23}\right)\right) \\
& \stackrel{(26)}{=} \lambda_{L E}\left(\left(\tau_{12}, \tau_{12}^{\prime \prime}, \beta_{12}^{\prime} \beta_{12}\right) \otimes\left(\tau_{23}, \tau_{23}^{\prime \prime}, \beta_{23}^{\prime} \beta_{23}\right)\right) \\
& \stackrel{(10)}{=} \lambda_{L E}\left(\mu\left(q_{12} \otimes q_{12}^{\prime}\right) \otimes \mu\left(q_{23} \otimes q_{23}^{\prime}\right)\right) .
\end{aligned}
$$

This shows that $\mu$ is fusion-preserving. Summarizing, we have defined an internal fusion product on the lifting bundle gerbe $\mathcal{G}_{L E}$.

Example 4.14 The spin lifting gerbe $\mathcal{S}_{L M}$ over the loop space of a spin manifold (see Example 4.3) is equipped with an internal fusion product.

If a bundle gerbe $\mathcal{G}$ is equipped with an internal fusion product $\lambda$, we can consider trivializations that "respect" the fusion product in the following way:

Definition 4.15 Let $\mathcal{G}$ be a bundle gerbe over $L M$ whose surjective submersion is the looping of a surjective submersion $\pi: Y \rightarrow M$. A fusion product on a trivialization $\mathcal{T}=(T, \kappa)$ of $\mathcal{G}$ is a fusion product $\lambda_{T}$ on the principal $A$-bundle $T$ over $L Y$. It is called compatible with an internal fusion product $\lambda$ on $\mathcal{G}$ if $\kappa$ is a fusion-preserving bundle morphism.

A morphism $\varphi:\left(T_{1}, \kappa_{1}, \lambda_{1}\right) \rightarrow\left(T_{2}, \kappa_{2}, \lambda_{2}\right)$ between trivializations with compatible fusion products is an ordinary morphism $\varphi:\left(T_{1}, \kappa_{1}\right) \rightarrow\left(T_{2}, \kappa_{2}\right)$ between the trivializations that is additionally fusion-preserving. The category of trivializations with compatible fusion product is denoted by $\mathcal{F} u s \operatorname{Triv}(\mathcal{G}, \lambda)$.

Theorem 4.16 The equivalence of Theorem 4.2 between trivializations of $\mathcal{G}_{L E}$ and $\mathcal{G}$-lifts of $L E$ extends to an equivalence in the fusion setting:

$$
\mathcal{F} u s \operatorname{Triv}\left(\mathcal{G}_{L E}, \lambda_{L E}\right) \cong \mathcal{G}-\mathcal{F} u s \mathcal{L} \text { ift }(L E) .
$$

Proof We recall that the equivalence of Theorem 4.2 sends a trivialization $(T, \kappa)$ to the principal $\mathcal{G}$-bundle $\mathcal{E}:=T$ with projection $T \rightarrow L E \rightarrow L M$ and $\mathcal{G}$-action given by

$$
q \cdot \beta:=\kappa\left(\beta^{-1} \otimes q\right) .
$$


The additional structure we want to take into account is the same on both sides: a fusion product $\lambda_{T}$ on the principal $A$-bundle $T \rightarrow L E$. It remains to check that the conditions are equivalent: on the left hand side the condition that $\kappa$ is fusion-preserving, and on the right hand side the condition that the $\mathcal{G}$-action on $T$ is fusion-preserving in the sense of Definition 4.11. Suppose first that $\kappa$ is fusion-preserving. Then

$$
\begin{aligned}
\lambda_{\varepsilon}\left(q_{12} \cdot \beta_{12} \otimes q_{23} \cdot \beta_{23}\right) & =\lambda_{\varepsilon}\left(\kappa\left(\beta_{12}^{-1} \otimes q_{12}\right) \otimes \kappa\left(\beta_{23}^{-1} \otimes q_{23}\right)\right) \\
& =\kappa\left(\lambda_{\mathcal{G}}\left(\beta_{12}^{-1} \otimes \beta_{23}^{-1}\right) \otimes \lambda_{\mathcal{E}}\left(q_{12} \otimes q_{23}\right)\right) \\
& =\kappa\left(\lambda_{\mathcal{G}}\left(\beta_{12} \otimes \beta_{23}\right)^{-1} \otimes \lambda_{\mathcal{E}}\left(q_{12} \otimes q_{23}\right)\right) \\
& =\lambda_{\varepsilon}\left(q_{12} \otimes q_{23}\right) \cdot \lambda_{\mathcal{G}}\left(\beta_{12} \otimes \beta_{23}\right) ;
\end{aligned}
$$

this shows that the $\mathcal{G}$-action is fusion-preserving. In the middle we have used that the multiplicativity (4) of $\lambda_{\mathcal{g}}$ implies

$$
\lambda_{\mathcal{G}}\left(\beta_{12} \otimes \beta_{23}\right)^{-1}=\lambda_{\mathcal{G}}\left(\beta_{12}^{-1} \otimes \beta_{23}^{-1}\right) .
$$

The converse can be proved similarly. The condition for morphisms is evidently the same on both sides.

Applied to fusion spin structures on loop spaces, Theorem 4.16 implies via Examples 4.12 and 4.14:

Corollary 4.17 There is an equivalence

$\{$ Fusion spin structures on $L M\} \cong\left\{\begin{array}{l}\text { Trivializations of the spin lifting gerbe } \\ \mathcal{S}_{L M} \text { with compatible fusion product }\end{array}\right\}$.

\section{Transgression of string structures}

In this section we prepare another important tool for the proof of our main result: we discuss string structures in the setting of bundle 2-gerbes.

\subsection{Bundle 2-gerbes and string structures}

We start with recalling some basic definitions.

Definition 5.1 [18, Definition 5.3] A bundle 2-gerbe over $M$ is a surjective submersion $\pi: Y \rightarrow M$ together with a bundle gerbe $\mathcal{P}$ over $Y^{[2]}$, an isomorphism

$$
\mathcal{M}: \operatorname{pr}_{12}^{*} \mathcal{P} \otimes \operatorname{pr}_{23}^{*} \mathcal{P} \rightarrow \operatorname{pr}_{13}^{*} \mathcal{P}
$$


of bundle gerbes over $Y^{[3]}$, and a transformation

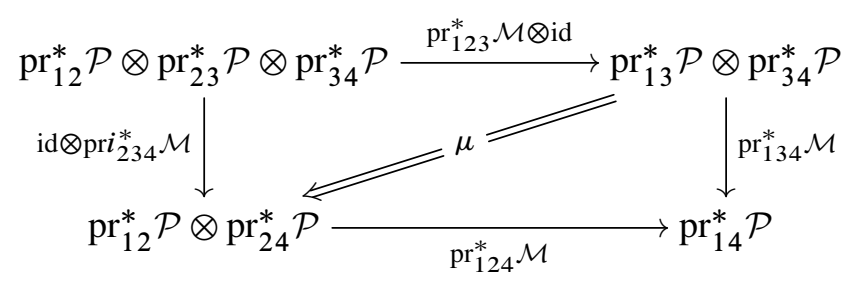

over $Y^{[4]}$ that satisfies the pentagon axiom.

The isomorphism $\mathcal{M}$ is called bundle 2 -gerbe product and the transformation $\mu$ is called associator. The pentagon axiom implies the cocycle condition for a certain degree three cocycle on $M$ with values in U(1). This, in turn, defines a class in $\mathrm{H}^{4}(M, \mathbb{Z})$; see [18, Proposition 7.2] for the details. For a bundle 2-gerbe $\mathbb{G}$, this class is denoted by $\operatorname{cc}(\mathbb{G}) \in \mathrm{H}^{4}(M, \mathbb{Z})$.

Definition 5.2 [18, Definition 11.1] Let $\mathbb{G}=(Y, \pi, \mathcal{P}, \mathcal{M}, \mu)$ be a bundle 2-gerbe over $M$. A trivialization of $\mathbb{G}$ is a bundle gerbe $\mathcal{S}$ over $Y$, together with a isomorphism

$$
\mathcal{A}: \mathcal{P} \otimes \operatorname{pr}_{2}^{*} \mathcal{S} \rightarrow \operatorname{pr}_{1}^{*} \mathcal{S}
$$

of bundle gerbes over $Y^{[2]}$ and a transformation

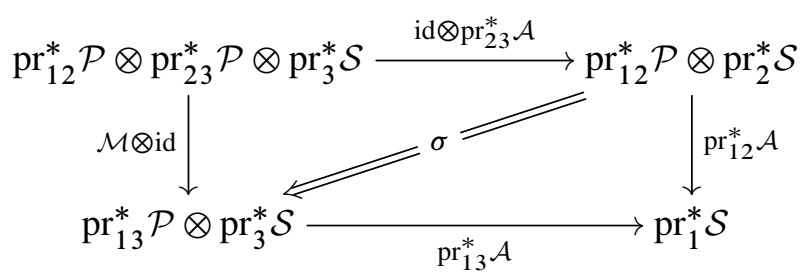

over $Y^{[3]}$ that satisfies a compatibility condition with the associator $\mu$.

As one expects, the characteristic class $\operatorname{cc}(\mathbb{G}) \in \mathrm{H}^{4}(M, \mathbb{Z})$ of $\mathbb{G}$ vanishes if and only if $\mathbb{G}$ admits a trivialization [18, Proposition 11.2]. An example of a bundle 2-gerbe that will be important later is the Chern-Simons 2-gerbe $\mathbb{C S}_{P}(\mathcal{G})$ [6]; it is associated to a principal $G$-bundle $P$ over $M$ and a multiplicative bundle gerbe $\mathcal{G}$ over $G$. Its construction is similar to the one of the lifting bundle gerbe in Section 4.1. We consider the difference map $\delta: P^{[2]} \rightarrow G$ of (9) and its generalization to higher fiber products, $\delta: P^{[k+1]} \rightarrow G^{k} \quad$ with $\quad\left(p_{1}, \ldots, p_{k}\right) \cdot \delta\left(p_{1}, \ldots, p_{k+1}\right)=\left(p_{2}, \ldots, p_{k+1}\right)$.

Now the Chern-Simons 2 -gerbe is obtained by pulling back the multiplicative bundle gerbe $\mathcal{G}$ along $\delta$; explicitly: 
- The surjective submersion is the bundle projection $P \rightarrow M$.

- The bundle gerbe $\mathcal{P}$ over $P^{[2]}$ is $\mathcal{P}:=\delta^{*} \mathcal{G}$.

- The bundle 2 -gerbe product is given by the pullback of the multiplicative structure (20):

$$
\delta^{*} \mathcal{M}: \operatorname{pr}_{12}^{*} \mathcal{P} \otimes \operatorname{pr}_{23}^{*} \mathcal{P} \rightarrow \operatorname{pr}_{13}^{*} \mathcal{P} .
$$

- The associator $\mu$ is given by the pullback of the transformation $\alpha$.

The similarity between the lifting bundle gerbe and the Chern-Simons 2-gerbe can be explained by the fact that the Chern-Simons 2-gerbe is the lifting 2-gerbe of a lifting problem for principal 2-bundles, or nonabelian gerbes; see [13, Theorem 7.1] and [14, Remark 4.2.4 and Theorem 4.2.7]. In the present context, the theory of nonabelian gerbes has two disadvantages that prevent us from using it here. The first is that it requires a strict 2-group model for the string group, which necessarily would be infinite-dimensional. The second disadvantage is that the only available formalism for the transgression of nonabelian gerbes (namely the one developed in [14]) is defined indirectly via the lifting bundle 2 -gerbe. In this article, we will avoid these difficulties and follow the approach of [28] to string structures.

For this purpose, we consider $P=F M$, the spin-oriented frame bundle of $M$, and $\mathcal{G}=\mathcal{G}_{\text {bas }}$, the basic bundle gerbe over $\operatorname{Spin}(n)$, whose Dixmier-Douady class is the fixed generator $\gamma_{\text {can }} \in \mathrm{H}^{3}(\operatorname{Spin}(n), \mathbb{Z})$. We write $\mathbb{C S}_{M}:=\mathbb{C S}_{P}\left(\mathcal{G}_{\text {bas }}\right)$ for the associated Chern-Simons 2-gerbe. We have [28, Theorem 1.1.3]

$$
\operatorname{cc}\left(\mathbb{C S}_{M}\right)=\frac{1}{2} p_{1}(M) .
$$

In particular, we see that $M$ is a string manifold if and only if $\mathbb{C S}_{M}$ admits a trivialization. This motivates the following definition:

Definition 5.3 [28, Definition 1.1.5] Let $M$ be a spin manifold. A string structure on $M$ is a trivialization of $\mathbb{C S}_{M}$.

Explicitly, a string structure on $M$ is a triple $(\mathcal{S}, \mathcal{A}, \sigma)$ consisting of a bundle gerbe $\mathcal{S}$ over $F M$, of an isomorphism

$$
\mathcal{A}: \delta^{*} \mathcal{G}_{\text {bas }} \otimes \operatorname{pr}_{2}^{*} \mathcal{S} \rightarrow \operatorname{pr}_{1}^{*} \mathcal{S}
$$

between bundle gerbes over $F M^{[2]}$, and of a transformation $\sigma$ over $F M^{[3]}$.

One can show [14, Section 6] that a string structure in the sense of Definition 5.3 is the same as a lift of the structure group of $F M$ to an infinite-dimensional, strict 
2-group model of the string group, in the framework of nonabelian gerbes (or principal 2-bundles).

Definition 5.3 of a string structure has many nice features, some of which are described in [28]. For example, it is completely finite-dimensional and does not involve the string group. A particular feature, which we need in the proof of the main theorem, is that Definition 5.3 reproduces the topological notion of a string class: a cohomology class $\xi \in \mathrm{H}^{3}(F M, \mathbb{Z})$ that restricts on each fiber to the generator $\gamma_{\text {can }} \in \mathrm{H}^{3}(\operatorname{Spin}(n), \mathbb{Z})$. Indeed, we have:

Proposition 5.4 [28, Theorem 1.1.4] The map

$\left\{\begin{array}{l}\text { Isomorphism classes of } \\ \text { string structures on } M\end{array}\right\} \rightarrow\{$ String classes on $F M\}, \quad(\mathcal{S}, \mathcal{A}, \sigma) \mapsto-\operatorname{dd}(\mathcal{S})$

that sends a trivialization to minus the Dixmier-Douady class of the bundle gerbe $\mathcal{S}$ is a bijection.

Let us recall one aspect of Proposition 5.4, namely the fact that the map is well-defined. This follows from the following lemma, which will be used later.

Lemma 5.5 Let $\mathcal{S}$ be a bundle gerbe over $F M$, and let $\mathcal{A}: \delta^{*} \mathcal{G}_{\text {bas }} \otimes \operatorname{pr}_{2}^{*} \mathcal{S} \rightarrow \operatorname{pr}_{1}^{*} \mathcal{S}$ be an isomorphism between bundle gerbes over $F M^{[2]}$. Then $-\operatorname{dd}(\mathcal{S})$ is a string class.

Proof Let $p \in F M$ be a point and $\iota_{p}: \operatorname{Spin}(n) \rightarrow F M, g \mapsto p g$ be the associated inclusion of the structure group of $F M$ in the fiber of $p$. Consider the map $s_{p}: \operatorname{Spin}(n) \rightarrow F M^{[2]}, g \mapsto(p, p g)$. It satisfies $\operatorname{pr}_{2} \circ s_{p}=\iota_{p}$ and $\delta \circ s_{p}=\mathrm{id}$, and $c_{p}:=\operatorname{pr}_{1} \circ s_{p}$ is the constant map with value $p$. We obtain an isomorphism

$$
s_{p}^{*} \mathcal{A}: \mathcal{G}_{\mathrm{bas}} \otimes \iota_{p}^{*} \mathcal{S} \rightarrow c_{p}^{*} \mathcal{S} .
$$

Since the pullback of a bundle gerbe along a constant map is trivial, it has vanishing Dixmier-Douady class. Thus, we get

$$
\gamma_{\text {can }}+\iota_{p}^{*} \operatorname{dd}(\mathcal{S})=0 .
$$

Hence, $-\operatorname{dd}(\mathcal{S})$ is a string class.

Another nice feature of Definition 5.3 is that it provides a basis to include string connections. The following definition summarizes the relevant notions concerning connections on bundle 2-gerbes.

Definition 5.6 Let $\mathbb{G}=(Y, \pi, \mathcal{P}, \mathcal{M}, \mu)$ be a bundle 2-gerbe over $M$. 
(i) A connection on $\mathbb{G}$ consists of a 3 -form $C \in \Omega^{3}(Y)$, and of a connection on the bundle gerbe $\mathcal{P}$ such that

$$
\operatorname{pr}_{2}^{*} C-\operatorname{pr}_{1}^{*} C=\operatorname{curv}(\mathcal{P}),
$$

and such that $\mathcal{M}$ and $\mu$ are connection-preserving.

(ii) Suppose $\mathbb{G}$ is equipped with a connection, and $\mathbb{T}=(\mathcal{S}, \mathcal{A}, \sigma)$ is a trivialization of $\mathbb{G}$. A compatible connection on $\mathbb{T}$ is a connection on the bundle gerbe $\mathcal{S}$ such that $\mathcal{A}$ and $\sigma$ are connection-preserving.

The Chern-Simons 2-gerbe $\mathbb{C S}_{P}(\mathcal{G})$ associated to a principal $G$-bundle $P$ over $M$ and a multiplicative bundle gerbe $\mathcal{G}$ over $G$ can be equipped with a connection that only depends on two parameters: a connection $A$ on the bundle $P$, and a multiplicative connection on the bundle gerbe $\mathcal{G}$, as recalled in Section 4.3, such that the two differential forms $H=\operatorname{curv}(\mathcal{G})$ and $\rho$ are the canonical ones; see (5) and (6). In the following we recall the data of the connection on the Chern-Simons 2-gerbe $\mathbb{C S}_{P}(\mathcal{G})$ on the basis of [22, Section 3.2].

- The 3-form is the Chern-Simons 3-form $C S(A) \in \Omega^{3}(P)$ associated to the connection $A$.

- The connection on the bundle gerbe $\mathcal{P}=\delta^{*} \mathcal{G}$ over $P^{[2]}$ is given by the connection on $\delta^{*} \mathcal{G}$ shifted by the 2 -form

$$
\omega:=-\left\langle\delta^{*} \bar{\theta} \wedge \operatorname{pr}_{1}^{*} A\right\rangle \in \Omega^{2}\left(P^{[2]}\right) .
$$

In other words, we have $\mathcal{P}=\delta^{*} \mathcal{G} \otimes \mathcal{I}_{\omega}$ as bundle gerbes with connection.

The required identity (27) is here a well-known identity in Chern-Simons theory:

$$
\operatorname{pr}_{2}^{*} C S(A)-\operatorname{pr}_{1}^{*} C S(A)=\delta^{*} H+\mathrm{d} \omega .
$$

This identity is the reason for the shift of the connection on $\delta^{*} \mathcal{G}$ by $\omega$; without that shift (27) would not hold. That the bundle 2 -gerbe product $\delta^{*} \mathcal{M}$ is connection-preserving for the shifted connections follows from the identity

$$
\delta^{*} \rho+\operatorname{pr}_{12}^{*} \omega-\operatorname{pr}_{13}^{*} \omega+\operatorname{pr}_{23}^{*} \omega=0
$$

of differential forms over $P^{[3]}$. Since the associator $\mu$ is just the pullback of the connection-preserving transformation $\alpha$, it is connection-preserving. This verifies that we have a connection on the Chern-Simons 2-gerbe $\mathbb{C S}_{P}(\mathcal{G})$.

In the situation of a spin manifold $M$, the Chern-Simons 2-gerbe $\mathbb{C S}_{M}=\mathbb{C S}_{F M}\left(\mathcal{G}_{\text {bas }}\right)$ carries a canonical connection, defined by the Levi-Civita connection on $M$ and the canonical connection on the basic bundle gerbe $\mathcal{G}_{\text {bas }}$; see [25, Theorem 1.2.1]. 
Definition 5.7 [28, Definition 1.2.2] Let $\mathbb{T}$ be a string structure. A string connection on $\mathbb{T}$ is a connection on $\mathbb{T}$ that is compatible with the canonical connection on $\mathbb{C S}_{M}$.

This concept of a string connection reproduces [28, Theorem 1.2.3] the original definition of Stolz and Teichner [19], formulated in terms of trivializations of Chern-Simons theory. One important statement about string connections that we will need later is the following theorem.

Theorem 5.8 [28, Theorem 1.3.4] Every string structure admits a string connection.

\subsection{Transgression}

We define transgression for (certain) bundle 2-gerbes and their trivializations. Let $\mathbb{G}$ be a bundle 2-gerbe with connection over $M$, consisting of a surjective submersion $\pi: Y \rightarrow M$, a curving $C \in \Omega^{3}(Y)$, a bundle gerbe $\mathcal{P}$ with connection over $Y^{[2]}$, and a connection-preserving bundle 2 -gerbe product $\mathcal{M}$ with connection-preserving associator $\mu$.

The restriction we impose on the admissible bundle 2-gerbes is that their surjective submersion $\pi: Y \rightarrow M$ is loopable, ie $L \pi: L Y \rightarrow L Y$ is again a surjective submersion (whereas in general $L \pi$ is not even surjective). For example, the projection of a principal bundle with connected structure group is loopable; see [23, Lemma 5.1]. For $\mathbb{G}$ a bundle 2-gerbe with loopable surjective submersion, we define the following bundle gerbe $\mathscr{T}_{\mathbb{G}}$ over $L M$. Its surjective submersion is $L \pi: L Y \rightarrow L M$. The principal $\mathrm{U}(1)$-bundle over $L Y^{[2]}$ is $\mathscr{T}_{\mathcal{P}}$. Since transgression for bundle gerbes is functorial, natural and monoidal, the transgression $\mathscr{T}_{\mathcal{M}}$ is a bundle gerbe product. It is associative since the associator $\mu$ of $\mathbb{G}$ transgresses to an equality.

The transgressed bundle gerbe $\mathscr{T}_{\mathbb{G}}$ over $L M$ carries a connection coming from the connections on the transgressed bundles. The curving this connection is $B:=-\int_{S^{1}} \mathrm{ev}^{*} C \in$ $\Omega^{2}(L Y)$, with the sign due to (16). As a consequence, we obtain the relation

$$
\operatorname{curv}\left(\mathscr{T}_{\mathbb{G}}\right)=-\int_{S^{1}} \operatorname{ev}^{*} \operatorname{curv}(\mathbb{G})
$$

Moreover, the transgression $\mathbb{G} \mapsto \mathscr{T}_{\mathbb{G}}$ fits well into the general context of transgression in differential cohomology.

Proposition 5.9 Under the transgression homomorphism $\widehat{\tau}: \widehat{\mathrm{H}}^{4}(M) \rightarrow \widehat{\mathrm{H}}^{3}(L M)$ in differential cohomology, we have

$$
\widehat{\tau}([\mathbb{G}])=-\left[\mathscr{T}_{\mathbb{G}}\right] .
$$


Proof Transgression in differential cohomology is characterized axiomatically; see [4, Definition 38]. One of the axioms is compatibility with the curvature. We conclude from $(29)$ that $\widehat{\tau}([\mathbb{G}])$ and $-\left[\mathscr{T}_{\mathbb{G}}\right]$ have the same curvature. Differential cohomology classes with the same curvature differ by an element in $\mathrm{H}^{2}(L M, \mathrm{U}(1)) \cong$ $\operatorname{Hom}\left(\mathrm{H}_{2}(L M), \mathrm{U}(1)\right)$. Every homology class in degree two is the image of the fundamental class of a closed oriented surface $\Sigma$ under a smooth map $\phi: \Sigma \rightarrow L M$. The element in $\operatorname{Hom}\left(\mathrm{H}_{2}(L M), \mathrm{U}(1)\right)$ is then given by the difference between the holonomies of $\widehat{\tau}([\mathbb{G}])$ and $-\left[\mathscr{T}_{\mathbb{G}}\right]$ along $\phi$. We prove in the following that these holonomies coincide; this shows the claim.

The homomorphism $\widehat{\tau}$ has the following property [4, Proposition 82]. Consider $\Phi: \Sigma \times$ $S^{1} \rightarrow M$ defined by $\Phi(s, z):=\phi(s)(z)$. Then $\operatorname{Hol}_{\widehat{\tau}([\mathbb{G}])}(\phi)=\operatorname{Hol}_{\mathbb{G}}(\Phi)$. We compute $\operatorname{Hol}_{\mathbb{G}}(\Phi)$ explicitly. The pullback $\Phi^{*} \mathbb{G}$ is trivializable for dimensional reasons. Its surjective submersion is $Z:=\left(\Sigma \times S^{1}\right)_{\Phi} \times_{\pi} Y$ with the projection $\pi_{Z}: Z \rightarrow \Sigma \times S^{1}$. We denote by $\operatorname{pr}_{Y}: Z \rightarrow Y$ the projection to $Y$. Let $\mathbb{T}$ be a trivialization of $\Phi^{*} \mathbb{G}$ with compatible connection, consisting of a bundle gerbe $\mathcal{S}$ with connection over $Z$, a connection-preserving isomorphism $\mathcal{A}: \operatorname{pr}_{Y}^{*} \mathcal{P} \otimes \operatorname{pr}_{2}^{*} \mathcal{S} \rightarrow \operatorname{pr}_{1}^{*} \mathcal{S}$ over $Z^{[2]}$, and of a connection-preserving transformation over $Z^{[3]}$. Let $K \in \Omega^{3}\left(\Sigma \times S^{1}\right)$ be the "covariant derivative" of the trivialization $\mathbb{T}$. It is determined by the condition

$$
\pi_{Z}^{*} K=\operatorname{pr}_{Y}^{*} C+\operatorname{curv}(\mathcal{S})
$$

By definition of holonomy, we have

$$
\operatorname{Hol}_{\mathbb{G}}(\Phi)=\exp \left(2 \pi \mathrm{i} \int_{\Sigma \times S^{1}} K\right)
$$

Next we compute $\operatorname{Hol}_{\mathscr{I}_{\mathcal{G}}}(\phi)$. We consider the pullback $\phi^{*} \mathscr{T}_{\mathbb{G}}$ to $\Sigma$. Its surjective submersion is $W:=\Sigma_{\phi} \times{ }_{L \pi} L Y$ with the projection $\pi_{W}: W \rightarrow \Sigma$. We define smooth maps $\alpha: \Sigma \rightarrow L\left(\Sigma \times S^{1}\right)$ by $\alpha(s)(z):=(s, z)$ and $\tilde{\alpha}: W \rightarrow L Z$ by $i(s, \tau)(z):=$ $(s, z, \tau(z))$. They fit into the commutative diagram

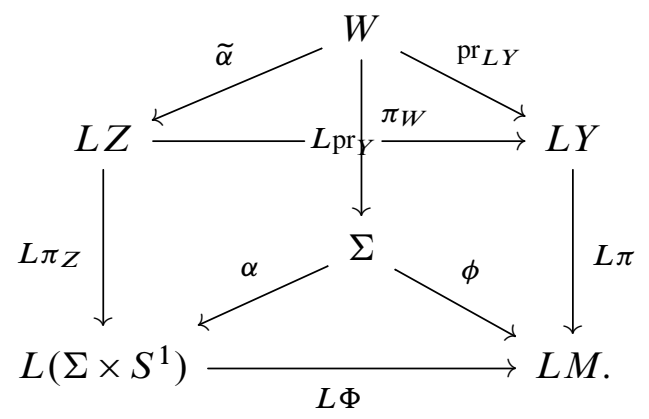


The map $\tilde{\alpha}$ extends to the fiber products, $\widetilde{\alpha}: W^{[k]} \rightarrow L Z^{[k]}$. We define a trivialization of $\phi^{*} \mathscr{T}_{\mathbb{G}}$ with compatible connection. It consists of the principal $\mathrm{U}(1)$-bundle $\tilde{\alpha}^{*} \mathscr{T}_{\mathcal{S}}$ over $W$ and of the connection-preserving isomorphism $\widetilde{\alpha}^{*} \mathcal{T}_{\mathcal{A}}$ over $W^{[2]}$. This trivialization is equipped with a compatible connection. Its covariant derivative is a 2 -form $\omega \in \Omega^{2}(\Sigma)$ determined by $\pi_{W}^{*} \omega=\operatorname{pr}_{L Y}^{*}\left(-\int_{S^{1}} C\right)+\operatorname{curv}\left(\widetilde{\alpha}^{*} \mathcal{T}_{\mathcal{S}}\right)$, ie the curving of $\phi^{*} \mathscr{T}_{\mathcal{G}}$ plus the curvature of the U(1)-bundle of its trivialization. Using (30) and the commutativity of (31), we conclude that $\omega=-\alpha^{*} \int_{S^{1}} \mathrm{ev}^{*} K$. This, in turn, is nothing but $\omega=-\int_{S^{1}} K$. We obtain

$\operatorname{Hol}_{\mathscr{J}_{\mathbb{G}}}(\phi)=\exp \left(2 \pi \mathrm{i} \int_{\Sigma} \omega\right)=\exp \left(-\int_{\Sigma \times S^{1}} K\right)=\operatorname{Hol}_{\mathbb{G}}(\Phi)^{-1}=\operatorname{Hol}_{\widehat{\tau}([\mathbb{G}])}(\phi)^{-1}$.

This shows that the holonomies of $\widehat{\tau}([\mathbb{G}])$ and $-\left[\mathscr{T}_{\mathbb{G}}\right]$ coincide.

Since the transgression homomorphism $\widehat{\tau}$ is compatible with the characteristic class, see [4, Proposition 47], we obtain:

\section{Corollary 5.10}

$$
\operatorname{dd}\left(\mathscr{T}_{\mathbb{G}}\right)=-\tau(\operatorname{cc}(\mathbb{G}))
$$

It is crucial for this article to observe that the transgressed bundle gerbe $\mathscr{T}_{\mathbb{G}}$ over $L M$ is equipped with an internal fusion product (Definition 4.13). Indeed, the principal $\mathrm{U}(1)$-bundle over $L Y^{[2]}$ in the structure of $\mathscr{T}_{\mathbb{G}}$, namely $\mathscr{T}_{\mathcal{P}}$, is the transgression of a bundle gerbe, and hence equipped with a fusion product. We denote this fusion product by $\lambda_{\mathbb{G}}$. Further, the bundle gerbe product of $\mathscr{T}_{\mathbb{G}}$, namely $\mathscr{T}_{\mathcal{M}}$, is the transgression of a bundle gerbe isomorphism, and hence fusion-preserving.

Next we discuss the transgression of trivializations of bundle 2-gerbes. If $\mathbb{T}=(\mathcal{S}, \mathcal{A}, \sigma)$ is a trivialization of $\mathbb{G}$ with compatible connection, we define a trivialization $\mathscr{T}_{\mathbb{T}}$ of $\mathscr{T}_{\mathbb{G}}$. The bundle gerbe $\mathcal{S}$ with connection transgresses to a principal U(1)-bundle $\mathscr{T}_{\mathcal{S}}$ over $L Y$. Since transgression is functorial, natural, and monoidal, the 1-morphism

$$
\mathcal{A}: \mathcal{P} \otimes \operatorname{pr}_{2}^{*} \mathcal{S} \rightarrow \operatorname{pr}_{1}^{*} \mathcal{S}
$$

over $Y^{[2]}$ transgresses to the required bundle morphism over $L Y^{[2]}$, and the 2isomorphism $\sigma$ implies the compatibility condition.

Since $\mathscr{T}_{\mathcal{S}}$ is a fusion bundle, the trivialization $\mathscr{T}_{\mathbb{T}}$ carries a fusion product, and since $\mathscr{T}_{\mathcal{A}}$ is fusion-preserving, it is compatible with the internal fusion product $\lambda_{\mathbb{G}}$ of $\mathscr{T}_{\mathbb{G}}$. We obtain a functor

$$
\mathscr{T}: \mathrm{h}_{1} \operatorname{Triv}^{\nabla}(\mathbb{G}) \rightarrow \mathcal{F} u s \operatorname{Triv}\left(\mathscr{T}_{\mathbb{G}}, \lambda_{\mathbb{G}}\right) .
$$

In the following we specialize to the Chern-Simons 2-gerbe and use the functor (32) to transgress (geometric) string structures. 
Theorem 5.11 [14, Proposition 6.2.1] Let $\mathcal{G}$ be a multiplicative bundle gerbe with connection over a Lie group $G$, and let $P$ be a principal $G$-bundle over $M$ with connection. Let $\mathbb{C S}_{P}(\mathcal{G})$ be the associated Chern-Simons 2-gerbe with connection. Let $\mathcal{G}_{L P}$ be the lifting gerbe associated to the problem of lifting the structure group of $L P$ from $L G$ to the central extension $\mathscr{T}_{\mathcal{G}}$. Then there is a canonical isomorphism

$$
\varphi: \mathscr{T}_{\mathbb{C S}_{P}(\mathcal{G})} \rightarrow \mathcal{G}_{L P}
$$

between bundle gerbes over $L M$.

The claimed isomorphism has been constructed in [14, Proposition 6.2.1]; since we need it explicitly below, we recall this construction. The main problem is to treat the occurrences of transgressions of trivial bundle gerbes, namely $\mathscr{T}_{\mathcal{I}_{\omega}}$ over $L P^{[2]}$ (coming from the shift in the connection on $\mathbb{C S}_{P}(\mathcal{G})$ ) and $\mathscr{I}_{\mathcal{I}_{\rho}}$ (coming from the multiplicative bundle gerbe $\mathcal{G}$ ). The construction proceeds as follows:

- Both bundle gerbes have the same surjective submersion, $L P \rightarrow L M$. In this situation, an isomorphism between bundle gerbes can be given by an isomorphism between the principal $\mathrm{U}(1)$-bundles over $L P^{[2]}$ that belong to the two bundle gerbes.

- Now we look at these principal U(1)-bundles over $L P^{[2]}$. The one of $\mathscr{T}_{\mathbb{C S}_{P}(\mathcal{G})}$ is $\mathscr{T}_{\mathcal{P}}$, where $\mathcal{P}=\delta^{*} \mathcal{G} \otimes \mathcal{I}_{\omega}$, while the one of $\mathcal{G}_{L P}$ is $P:=L \delta^{*} \mathscr{T}_{\mathcal{G}}$. Naturality of transgression and the canonical trivialization $t_{\omega}$ of $\mathscr{I}_{\mathcal{I}_{\omega}}$ provide an isomorphism

$$
\mathscr{T}_{\mathcal{P}} \cong L \delta^{*} \mathscr{T}_{\mathcal{G}} \otimes \mathscr{T}_{\mathcal{I}_{\omega}} \stackrel{\operatorname{id} \otimes t_{\omega}}{\longrightarrow} L \delta^{*} \mathscr{T}_{\mathcal{G}}=P
$$

between principal $\mathrm{U}(1)$-bundles over $L P^{[2]}$; this gives all the data for the isomorphism $\varphi$ we want to construct

- It remains to check that $\varphi: \mathscr{T}_{\mathcal{P}} \rightarrow P$ commutes with the bundle gerbes products of $\mathscr{T}_{\mathbb{C S}_{P}(\mathcal{G})}$ and $\mathcal{G}_{L P}$ in the sense that the diagram

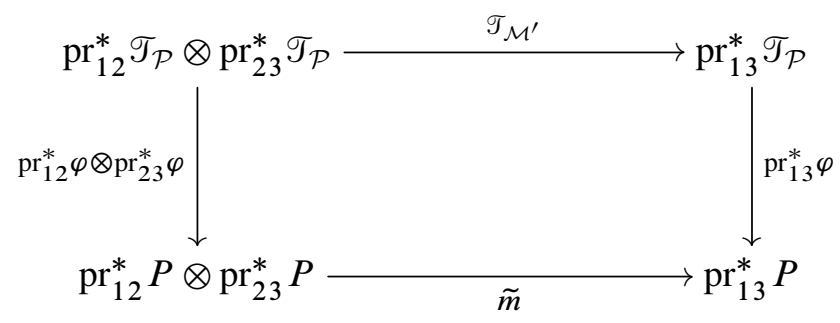


is commutative. Indeed, substituting the definitions gives the diagram

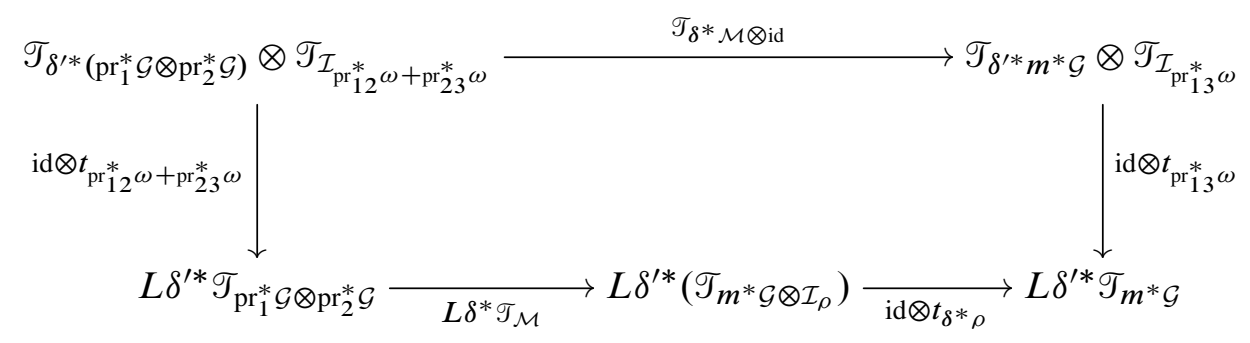

which is commutative due to the identity (28).

Summarizing, we have constructed an isomorphism $\varphi: \mathscr{T}_{\mathbb{C S}_{P}(\mathcal{G})} \rightarrow \mathcal{G}_{L P}$ of bundle gerbes over $L M$.

In the situation of a spin manifold $M$ we obtain an isomorphism

$$
\varphi: \mathscr{T}_{\mathbb{C S}_{M}} \rightarrow \mathcal{S}_{L M}
$$

between the transgression of the Chern-Simons 2-gerbe $\mathbb{C S}_{M}=\mathbb{C S}_{F M}\left(\mathcal{G}_{\text {bas }}\right)$ and the spin lifting gerbe $\mathcal{S}_{L M}$; see Example 4.3. With Corollary 5.10, we reproduce the result of [10] mentioned in the introduction:

$$
\tau\left(\frac{1}{2} p_{1}(M)\right)=\lambda_{L M}
$$

Next we come to a new, crucial property of the isomorphism $\varphi$.

Proposition 5.12 The isomorphism $\varphi$ of Theorem 5.11 respects the internal fusion products.

Proof We recall that the internal fusion product of $\mathscr{T}_{\mathbb{C S}_{P}}(\mathcal{G})$ is the fusion product $\lambda_{\mathcal{P}}$ on the $\mathrm{U}(1)$-bundle $\mathscr{T}_{\mathcal{P}}$, while the fusion product of $\mathcal{G}_{L P}$ is the pullback of the fusion product $\lambda_{\mathcal{G}}$ of $\mathscr{T}_{\mathcal{G}}$ along $L \delta$. The claim is that $\varphi$, ie the map

$$
\mathscr{T}_{\mathcal{P}} \cong L \delta^{*} \mathscr{T}_{\mathcal{G}} \otimes \mathscr{T}_{\mathcal{I}_{\omega}} \stackrel{\operatorname{id} \otimes t_{\omega}}{\longrightarrow} L \delta^{*} \mathscr{T}_{\mathcal{G}}=P
$$

is fusion-preserving. The canonical bundle morphism $\mathscr{T}_{\mathcal{P}} \cong L \delta^{*} \mathscr{T}_{\mathcal{G}} \otimes \mathscr{T}_{\mathcal{I}_{\omega}}$ induced by the naturality of the transgression functor $\mathscr{T}$ is fusion-preserving, and by [23, Lemma 3.6] the trivialization $t_{\omega}$ is fusion-preserving. Thus $\varphi$ is a composition of fusion-preserving isomorphisms, and hence fusion-preserving. 


\section{Proof of Theorem 1.4}

In this section we prove the main theorem of this article: the assertion that $M$ is string if and only if $L M$ is fusion spin. By Corollary 4.17, $L M$ is fusion spin if and only if the spin lifting gerbe $\mathcal{S}_{L M}$ has a trivialization with compatible fusion product.

Suppose first that $M$ is string, so that there exists a string structure $\mathbb{T}$ (Definition 5.3). By Theorem $5.8 \mathbb{T}$ admits a string connection, together giving a trivialization of $\mathbb{C S}_{M}$ with compatible connection. Its transgression is a trivialization $\mathscr{T}_{\mathbb{T}}$ of $\mathscr{T}_{\mathbb{C S}_{M}}$ with compatible fusion product; see Section 5.2. Since the isomorphism $\mathscr{T}_{\mathbb{C S}_{M}} \cong \mathcal{S}_{L M}$ of Theorem 5.11 preserves the internal fusion products (Proposition 5.12), $\mathscr{T}_{\mathbb{T}}$ induces a trivialization of $\mathcal{S}_{L M}$ with compatible fusion product.

Conversely, suppose $\mathcal{S}_{L M}$ has a trivialization $(T, \kappa)$ with compatible fusion product $\lambda_{T}$. Let $p \in F M$ be a point. We may assume that $M$ is connected, otherwise we proceed with each connected component of $M$ separately. Then $F M$ is also connected. Thus we have a well-defined regression functor

$$
\mathscr{R}_{p}: \mathcal{F} \operatorname{usBun}(L F M) \rightarrow \mathcal{G r b}(F M)
$$

and obtain a bundle gerbe $\mathcal{S}:=\mathscr{R}_{p}\left(T, \lambda_{T}\right)$ over $F M$. In $F M^{[2]}$ we choose the base point $(p, p)$. Then both projections $\operatorname{pr}_{1}, \operatorname{pr}_{2}: F M^{[2]} \rightarrow F M$ are base pointpreserving. Now, the fusion-preserving bundle morphism $\kappa$ over $L F M^{[2]}$ regresses to an isomorphism

$$
\mathscr{R}_{(p, p)}\left(P \otimes \mathrm{pr}_{2}^{*} T\right) \rightarrow \mathscr{R}_{(p, p)}\left(\mathrm{pr}_{1}^{*} T\right)
$$

between bundle gerbes over $F M^{[2]}$, where $P$ is the principal U(1)-bundle of the spin lifting gerbe. Going through its construction using the model $\overline{L \operatorname{Spin}(n)}=\mathscr{T}_{\mathcal{G}_{\text {bas }}}$, we find that $P=L \delta^{*} \mathcal{T}_{\mathcal{G}_{\text {bas }}}$. Then we use that there is a (canonical) isomorphism $\mathscr{R}_{1}\left(\mathscr{T}_{\mathcal{G}_{\text {bas }}}\right) \cong \mathcal{G}_{\text {bas }}$ (see Theorem 4.6). We get an isomorphism

$$
\mathscr{R}_{(p, p)}(P)=\mathscr{R}_{(p, p)}\left(L \delta^{*} \mathcal{T}_{\mathcal{G}_{\text {bas }}}\right)=\delta^{*} \mathscr{R}_{1}\left(\mathcal{T}_{\mathcal{G}_{\text {bas }}}\right) \cong \delta^{*} \mathcal{G}_{\text {bas }}
$$

Using that regression is monoidal and natural (see Lemma 4.4), we end up with an isomorphism

$$
\mathcal{A}: \delta^{*} \mathcal{G}_{\mathrm{bas}} \otimes \operatorname{pr}_{2}^{*} \mathcal{S} \rightarrow \operatorname{pr}_{1}^{*} \mathcal{S} .
$$

By Lemma $5.5-\mathrm{dd}(\mathcal{S})$ is a string class; thus, $M$ is string.

\section{References}

[1] O Alvarez, T P Killingback, M Mangano, P Windey, The Dirac-Ramond operator in string theory and loop space index theorems, Nuclear Phys. B Proc. Suppl. 1A (1987) 189-215 MR1005560 
[2] O Alvarez, T P Killingback, M Mangano, P Windey, String theory and loop space index theorems, Comm. Math. Phys. 111 (1987) 1-10 MR896755

[3] M F Atiyah, Circular symmetry and stationary-phase approximation, from: "Colloquium in honor of Laurent Schwartz, Vol. 1”, Astérisque 131 (1985) 43-59 MR816738

[4] C Bär, C Becker, Differential characters, Lecture Notes in Mathematics 2112, Springer, Berlin (2014) MR3237728

[5] J-L Brylinski, D A McLaughlin, The geometry of degree-four characteristic classes and of line bundles on loop spaces, I, Duke Math. J. 75 (1994) 603-638 MR1291698

[6] A L Carey, S Johnson, M K Murray, D Stevenson, B-L Wang, Bundle gerbes for Chern-Simons and Wess-Zumino-Witten theories, Comm. Math. Phys. 259 (2005) 577-613 MR2174418

[7] A L Carey, M K Murray, String structures and the path fibration of a group, Comm. Math. Phys. 141 (1991) 441-452 MR1134932

[8] T P Killingback, World-sheet anomalies and loop geometry, Nuclear Phys. B 288 (1987) 578-588 MR892061

[9] K Kuribayashi, T Yamaguchi, The vanishing problem of the string class with degree 3, J. Austral. Math. Soc. Ser. A 65 (1998) 129-142 MR1646609

[10] D A McLaughlin, Orientation and string structures on loop space, Pacific J. Math. 155 (1992) 143-156 MR1174481

[11] J Mickelsson, Kac-Moody groups, topology of the Dirac determinant bundle, and fermionization, Comm. Math. Phys. 110 (1987) 173-183 MR887993

[12] M K Murray, Bundle gerbes, J. London Math. Soc. 54 (1996) 403-416

[13] T Nikolaus, K Waldorf, Four equivalent versions of nonabelian gerbes, Pacific J. Math. 264 (2013) 355-419 MR3089401

[14] T Nikolaus, K Waldorf, Lifting problems and transgression for non-abelian gerbes, Adv. Math. 242 (2013) 50-79 MR3055987

[15] K Pilch, N P Warner, String structures and the index of the Dirac-Ramond operator on orbifolds, Comm. Math. Phys. 115 (1988) 191-212 MR931661

[16] A Pressley, G Segal, Loop groups, Oxford Univ. Press, Oxford (1986) MR900587

[17] M Spera, T Wurzbacher, Twistor spaces and spinors over loop spaces, Math. Ann. 338 (2007) 801-843 MR2317752

[18] D Stevenson, Bundle 2-gerbes, Proc. London Math. Soc. 88 (2004) 405-435

[19] S Stolz, P Teichner, What is an elliptic object?, from: "Topology, geometry and quantum field theory”, (U Tillmann, editor), London Math. Soc. Lecture Note Ser. 308, Cambridge Univ. Press, Cambridge (2004) 247-343 MR2079378 
[20] S Stolz, P Teichner, The spinor bundle on loop spaces (2005) preprint Available at http://people.mpim-bonn.mpg.de/teichner/Math/Surveys_files/ MPI. pdf

[21] K Waldorf, More morphisms between bundle gerbes, Theory Appl. Categ. 18 (2007) 240-273 MR2318389

[22] K Waldorf, Multiplicative bundle gerbes with connection, Differential Geom. Appl. 28 (2010) 313-340 MR2610397

[23] K Waldorf, A loop space formulation for geometric lifting problems, J. Aust. Math. Soc. 90 (2011) 129-144 MR2810948

[24] K Waldorf, A construction of string 2-group models using a transgression-regression technique, from: "Analysis, geometry and quantum field theory", (C L Aldana, M Braverman, B Iochum, C Neira Jiménez, editors), Contemp. Math. 584, Amer. Math. Soc., Providence, NJ (2012) 99-115 MR3013040

[25] K Waldorf, Transgression to loop spaces and its inverse, I: Diffeological bundles and fusion maps, Cah. Topol. Géom. Différ. Catég. 53 (2012) 162-210 MR2905777

[26] K Waldorf, Transgression to loop spaces and its inverse, II: Gerbes and fusion bundles with connection, preprint (2013) arXiv:1004.0031

[27] K Waldorf, Transgression to loop spaces and its inverse, III: Gerbes and thin fusion bundles, Adv. Math. 231 (2012) 3445-3472 MR2980505

[28] K Waldorf, String connections and Chern-Simons theory, Trans. Amer. Math. Soc. 365 (2013) 4393-4432 MR3055700

[29] E Witten, The index of the Dirac operator in loop space, from: "Elliptic curves and modular forms in algebraic topology", (P S Landweber, editor), Lecture Notes in Math. 1326, Springer, Berlin (1988) 161-181 MR970288

Institut für Mathematik und Informatik, Ernst-Moritz-Arndt-Universität Greifswald Walther-Rathenau-Str. 47, D-17493 Greifswald, Germany

konrad.waldorf@uni-greifswald.de

http://waldorf .math-inf.uni-greifswald.de

Received: 1 December 2013 Revised: 10 April 2015 
